\title{
Parametric analysis of nightside conductance effects on inner magnetospheric dynamics for the 17 April 2002 storm
}

\author{
Michael W. Liemohn, ${ }^{1}$ Aaron J. Ridley, ${ }^{1}$ Pontus C. Brandt, ${ }^{2}$ Dennis L. Gallagher, ${ }^{3}$ \\ Janet U. Kozyra, ${ }^{1}$ Daniel M. Ober, ${ }^{4}$ Donald G. Mitchell, ${ }^{2}$ Edmond C. Roelof, ${ }^{2}$ \\ and Robert DeMajistre ${ }^{2}$
}

Received 28 February 2005; revised 21 June 2005; accepted 27 June 2005; published 15 December 2005.

[1] Numerous simulations were conducted in order to quantify the influence of nightside conductance morphology and intensity on the storm-time ring current and plasmasphere. The study focused on the moderate magnetic storm of 17 April 2002. The simulation results were compared against measurements of the ring current and plasmasphere in order to assess the accuracy as a function of conductance parameter setting. In particular, three data sets were used: $D s t^{*}$, plasmapause location as extracted from IMAGE EUV snapshots, and IMAGE HENA flux observations for the 39-60 keV energy range. While no single simulation conducted for this study proved itself to be the best overall match to the selected data sets, many things were learned from the simulations. The most important scientific finding is that there is an optimal conductance level for maximal ring current intensity. Too little conductance leads to large shielding potentials that effectively inhibit ring current growth, while too much conductance leads to continual flow-through of the hot ions with no significant hot ion accumulation in the inner magnetosphere. It was found that the poleward shift of the high conductance region of the auroral oval was the most important factor affecting the data-model comparisons.

The peak intensity of the auroral oval conductance was also determined to be a significant factor affecting model accuracy. Tilting the dawnside location of the oval equatorward with respect to its duskside latitude had little effect on the results, as did the setting for the uniformly applied baseline conductance. The high-latitude boundary condition for the potential solution was also found to have little influence on the results.

Citation: Liemohn, M. W., A. J. Ridley, P. C. Brandt, D. L. Gallagher, J. U. Kozyra, D. M. Ober, D. G. Mitchell, E. C. Roelof, and R. DeMajistre (2005), Parametric analysis of nightside conductance effects on inner magnetospheric dynamics for the 17 April 2002 storm, J. Geophys. Res., 110, A12S22, doi:10.1029/2005JA011109.

\section{Introduction}

[2] A primary determinant for inner magnetospheric plasma dynamics is the electric field, which heavily influences the flow of charged particles. Because the inner magnetosphere is often assumed to be free of large parallel potential differences, the electric field pattern in the equatorial plane can be modeled as a simple mapping of the midlatitude ionospheric potential pattern along the magnetic field (B) lines. The two largest factors in specifying this potential pattern are the field-aligned currents (FACs) into and out of the ionosphere in this region (the sources and

\footnotetext{
${ }^{1}$ Department of Atmospheric, Oceanic, and Space Sciences, University of Michigan, Ann Arbor, Michigan, USA.

${ }^{2}$ Johns Hopkins University Applied Physics Laboratory, Laurel, Maryland, USA.

${ }^{3}$ National Space Science and Technology Center, NASA Marshall Space Flight Center, Huntsville, Alabama, USA.

${ }^{4}$ ATK Mission Research, Nashua, New Hampshire, USA.

Copyright 2005 by the American Geophysical Union. 0148-0227/05/2005JA011109
}

sinks) and the ionospheric conductance $(\Sigma)$ in this region (regulating the cross-B path of the current).

[3] This chain reveals that ionospheric conductance plays a critical role in determining the formation and evolution of the inner magnetospheric plasma populations, in particular the plasmasphere and the ring current. The plasmasphere is the cold, dense population in a torus extending a few Earth radii $\left(R_{E}\right)$ out from the planet [e.g., Lemaire and Gringauz, 1998]. Its characteristic temperature of a few eV or less means that these particles experience essentially no magnetic drifts and convection and corotation electric fields control their motion through space. Its high density is formed with the help of corotation dominance near the Earth, extending farther out during quiet times, allowing the magnetic flux tubes to fill with ionospheric plasma. This takes several days [Carpenter and Anderson, 1992], and a sharp boundary (a steep density gradient known as the plasmapause) forms between the corotation and convection dominated regions. When a magnetic storm occurs, the convection electric field rapidly strengthens, stripping away the outer plasmasphere and forming a drainage plume extending to the dayside magnetopause. When convection 
relaxes, the corotation-dominated region expands again and the plume within this new limit wraps up around the shrunken storm-time plasmasphere. Throughout this process, small-scale perturbations in the convection electric field distort the morphology of the plasmasphere and plume.

[4] The ring current is created by hot ions and electrons in the inner magnetosphere with energies between a few and a few hundred $\mathrm{keV}$ (Frank [1970] and the recent reviews by Daglis et al. [1999] and Ebihara and Ejiri [2002]). It is interesting that Singer [1957] suggested the ring current concept before the spaceflight era, as a hypothesis for explaining the ground-based magnetic perturbations during storms. Parker [1957] then detailed the formulation of this connection between magnetospheric particles and groundbased magnetic perturbations. The magnetic gradient and curvature drifts that influence these particles send the ions westward around Earth and the electrons eastward. When the known morphology of pressure gradients are considered, the result is two rings of current around the planet, a weak eastward current inside of a stronger westward current. In quiet times, these rings are approximately symmetric in local time, usually with a total net westward current of less than $1 \mathrm{MA}$, located a few $R_{E}$ away from Earth [e.g., Tsyganenko, 2000; Jorgensen et al., 2004]. During active times, the convection electric field (as well as inductive fields from magnetic reconfigurations) injects plasma sheet plasma into the inner magnetosphere, pumping up the total current up to several megaamps, perhaps as high as 15 MA during the most severe storms [e.g., Iyemori, 1990; Liemohn et al., 2001; Jorgensen et al., 2004]. The current is not symmetric during this period but rather highly asymmetric [e.g., Grafe, 1999; Greenspan and Hamilton, 2000; Liemohn et al., 2001]. The presence of so much hot plasma in the inner magnetosphere, however, is not a stable state, and the resulting electric potential pattern (from the field-aligned currents at the eastward and westward ends of the pressure bulge) tries to eject the particles from near-Earth space [e.g., Jaggi and Wolf, 1973; Liemohn and Brandt, 2005]. Therefore the morphology of this partial ring current is highly dependent on the midlatitude ionospheric conductance, which determines the large- and small-scale structure of the electric potential pattern.

[5] Early studies of the storm-time inner magnetosphere showed that the electric potential distribution can be quite distorted from a simplistic two-cell convection pattern [e.g., Southwood and Wolf, 1978]. Recent studies have tried to quantify this distortion [e.g., Fok et al., 2001, 2003; Ridley and Liemohn, 2002; Chen et al., 2003], finding that the storm-time partial ring current creates a large potential well near midnight and strong electric fields in the $+y_{\mathrm{GSM}}$ direction in the dusk and evening sectors. Sazykin et al. [2002] found that small potential vortices form when a low-density plasma sheet is injected in right behind a high-density injection, causing an interchange instability that mixes the two plasma regimes. The formation and persistence of these vortices depends on FACs pouring into a region of low ionospheric conductance. Khazanov et al. [2003] found that adding the conductance from the precipitating hot ions (in addition to the hot electrons, which is the standard assumption for auroral oval conductance) causes significant alterations to the potential pattern. Garner [2003] conducted numerical experiments to determine the relationship between the near-Earth electric potential pattern and the plasma sheet characteristics, finding that shielding is strong when the plasma is cold and dense but weak when the plasma is hot and rarified. Ebihara et al. [2005] found that there is a nonlinear relationship between the resulting ring current strength and the incident plasma sheet density. This nonlinearity arises because of the feedback loop between the partial ring current and the potential pattern and is modulated by the conductance.

[6] At most, these studies have compared two conductance models. Ebihara et al. [2004] and Ebihara and Fok [2004] are the only recent studies to systematically investigate the influence of conductance on the development of the storm-time ring current. These studies, however, only showed variations of one or two parameters controlling the nightside conductance.

[7] An extensive and systematic investigation of the influence of the nightside subauroral conductance pattern on the morphology and dynamics of the electric potential in the inner magnetosphere (and its influence on the ring current and plasmasphere) has not been conducted. Therefore it is useful to conduct large-scale parametric study on the influence of the location and strength of the auroral oval conductances relative to the FACs from the storm-time partial ring current. Such a study is presented here for the 17 April 2002 magnetic storm. Several data sets are used to quantify the accuracy of the simulation results, and the influence of each parameter on the goodness of fit is discussed and analyzed.

\section{The 17 April 2002 Magnetic Storm}

[8] Around 1200 UT on 17 April 2002, a week-long torrent of solar wind disturbances against the Earth's magnetosphere began. The first magnetic storm, on 17 April, was caused by the passage of the high solar wind density sheath preceding the magnetic cloud, and the subsequent low solar wind density magnetic cloud caused a second storm on 18 April.

[9] Figure 1 summarizes the geophysical and solar wind conditions on 17 April. Figure 1a shows the Dst index [Sugiura and Kamei, 1991] and its derivative index Dst*. $D s t^{*}$ is defined here as Dst with the removal of the magnetopause current influence, a quiet time offset, and a factor accounting for the induced currents inside the Earth (see equation (2) of Liemohn and Kozyra [2003] for the exact $D s t^{*}$ formula used in this study). $D s t^{*}$ reaches nearly $-120 \mathrm{nT}$ at $1700 \mathrm{UT}$ on 17 April and recovers to about $-50 \mathrm{nT}$ by the end of the day.

[10] Figure $1 \mathrm{~b}$ presents the solar wind dynamic pressure $\left(P_{\mathrm{SW}}\right)$ as observed by the SWEPAM instrument on the ACE spacecraft [McComas et al., 1998]. There is no data prior to $\sim 0800$ UT, but this gap does not affect this study. The dashed line shows the presumed values for this interval, which are consistent with the steady and nominal solar wind values observed by Wind (at $y_{\mathrm{GSM}} \sim 200 R_{E}$ ) and Geotail (in the magnetosheath). It is seen that the sheath was led by a large pressure pulse of over $20 \mathrm{nPa}$, followed by highly variable dynamic pressure that slowly decreases to less than $5 \mathrm{nPa}$ by the end of the day. Note that these data (as well as 


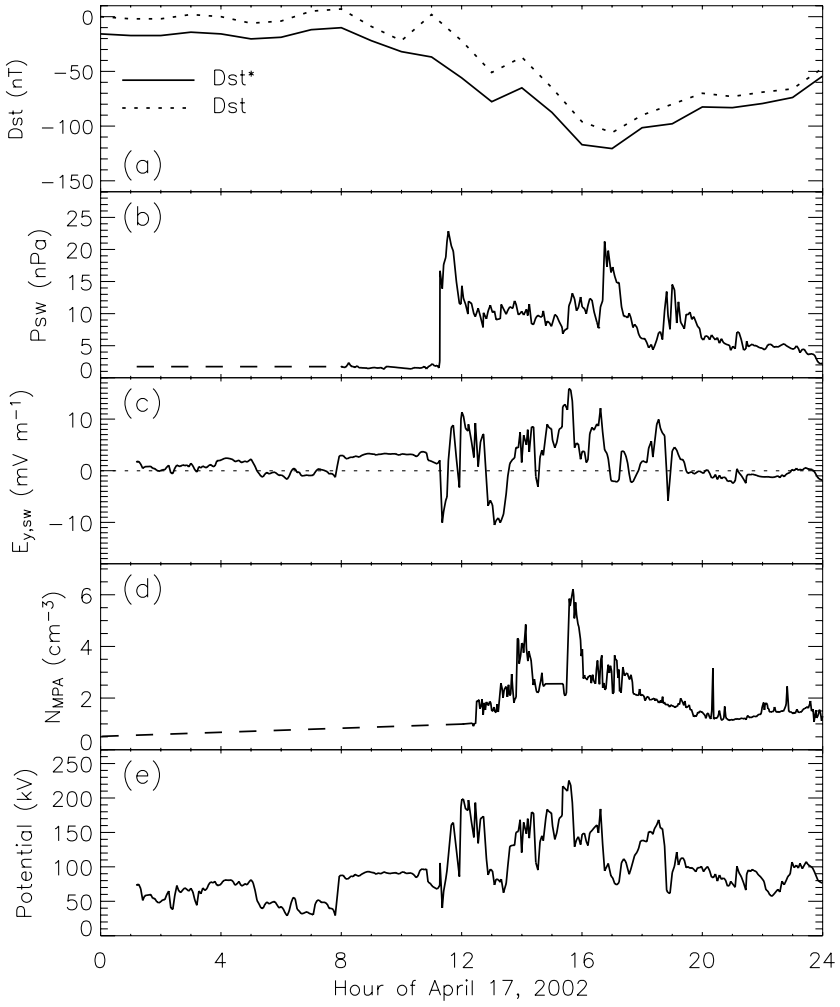

Figure 1. Geophysical quantities for 17 April 2002. Shown are (a) Dst and Dst*, (b) solar wind dynamic pressure, (c) solar wind motional electric field, (d) nightside hot ion density as measured by the MPA instruments, and (e) cross polar cap potential from the Weimer model.

the rest of the ACE measurements) have been shifted to the Earth using a $\Delta x_{\mathrm{GSM}} / v_{\mathrm{x}, \mathrm{GSM}}$ time delay. While more sophisticated solar wind propagation techniques exist [e.g., Weimer et al., 2003], the exact timing of the solar wind (that is, better than a few minutes error) is not a particularly critical factor when modeling a 12-hour storm event.

[11] In Figure $1 \mathrm{c}$ is the $y_{\mathrm{GSM}}$ component of the solar wind motional electric field $\left(\mathrm{E}_{\mathrm{y}, \mathrm{SW}}\right)$ which is the product of $v_{\mathrm{x}, \mathrm{GSM}}$ from SWEPAM and the $z_{\mathrm{GSM}}$ component of the interplanetary magnetic field (IMF), as measured by ACE MFE [Smith et al., 1998]. Positive values correspond to southward IMF, and $E_{\mathrm{y}, \mathrm{Sw}}$ exceeds $10 \mathrm{mV} / \mathrm{m}$ several times during the sheath passage.

[12] Figure 1d shows the near-Earth plasma sheet density as seen by the magnetospheric plasma analyzer (MPA) instrument on the geosynchronous spacecraft operated by the Los Alamos National Laboratory [Bame et al., 1993]. This value $\left(N_{\text {MPA }}\right)$ is a composite from all of the available MPA instruments (i.e., from several satellites), selecting the maximum density seen by any satellite on the nightside half of the magnetosphere (see Liemohn et al. [2001] for details on the data selection criteria). Note that there is no MPA data before $\sim 1200 \mathrm{UT}$, but this is before the storm and does not significantly affect the results of this study. $N_{\text {MPA }}$ quickly rises from below $1 \mathrm{~cm}^{-3}$ to $5 \mathrm{~cm}^{-3}$ and eventually exceeds $6 \mathrm{~cm}^{-3}$ during the sheath passage. It drops back down below $2 \mathrm{~cm}^{-3}$ by the end of the day.
[13] Figure 1e presents the cross polar cap potential difference for this day as computed from the Weimer [1996] empirical model. The potential difference is below $100 \mathrm{kV}$ before the storm and then jumps up to nearly $200 \mathrm{kV}$ soon after the shock arrival. It widely varies (from below $100 \mathrm{kV}$ to greater than $200 \mathrm{kV}$ ) for the rest of the day and eventually reduces to near $100 \mathrm{kV}$ by 2400 UT.

\section{Numerical Approach}

[14] The numerical tool to be employed for this parametric study of the nightside conductance effects on the stormtime inner magnetosphere is the ring current-atmosphere interaction model (RAM). RAM solves the gyration and bounce-averaged Boltzmann equation inside of some selected radial distance using second-order accurate numerical schemes to get the phase space density of any number of hot plasma species throughout the inner magnetosphere. Developed at the University of Michigan [Fok et al., 1993; Jordanova et al., 1996], there are now several branches of this model currently in use, namely those at NASA Goddard Space Flight Center [Fok et al., 2003; Ebihara et al., 2004, 2005], the University of New Hampshire [Jordanova et al., 2003], NASA Marshall Space Flight Center [Khazanov et al., 2003], and the University of Michigan [Kozyra et al., 2002; Liemohn et al., 2004].

[15] The specific version of RAM to be used here is that described by Liemohn et al. [2004]. The hot ion simulation domain is from $L=1.75$ to 6.75 . The grid in velocity space covers the full range of the ring current in energy $(10 \mathrm{eV}$ to $400 \mathrm{keV})$ and equatorial pitch angle $\left(0\right.$ to $\left.90^{\circ}\right)$. RAM uses geosynchronous plasma observations from the spacecraft

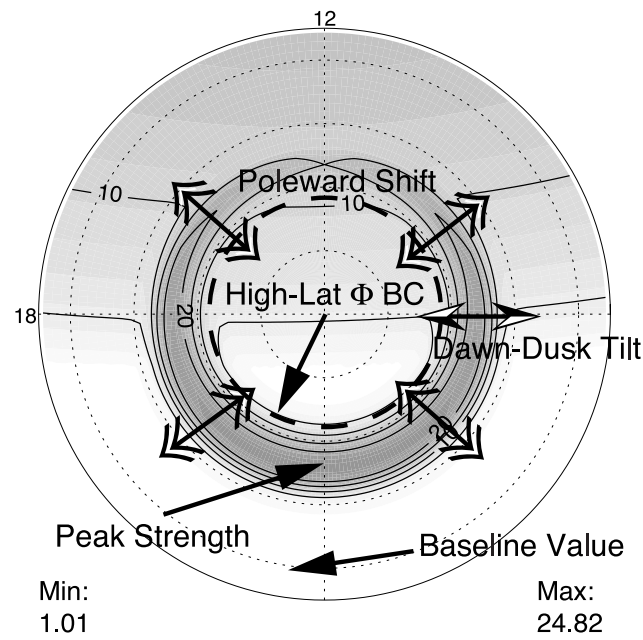

Figure 2. Example ionospheric Pedersen conductance pattern, annotated to show the various parameters to be varied in this study. The view is over the north magnetic pole with noon to the top, and thin dotted lines are drawn every $10^{\circ}$ magnetic latitude. The arrows with the doubleline heads at each end show the poleward shift, the arrow with the black-and-white heads at each end shows the dawn-dusk tilt, and the solid-head arrows point from the label to the region being varied. The maximum and minimum values of $\Sigma_{\mathrm{P}}$ in this plot are listed in the bottom corners. 
operated by Los Alamos National Laboratory (LANL), namely the data from the magnetospheric plasma analyzer (MPA) [Bame et al., 1993] and the synchronous orbiting particle analyzer (SOPA) [Belian et al., 1992]. Ion composition $\left(\mathrm{H}^{+}\right.$and $\mathrm{O}^{+}$are used in this study) is specified using the Young et al. [1982] relationship, with the methodology described by Liemohn et al. [1999, 2001]. The magnetic field is a static dipole, which is assumed a reasonable approximation for this moderately sized storm. The hydrogen geocorona used for charge exchange loss is that of Rairden et al. [1986]. RAM is actively coupled, with feedback, to the dynamic global core plasma model (DGCPM) of Ober et al. [1997], for the thermal plasma description in the inner magnetosphere.

[16] The model used here is the version described by Liemohn et al. [2004] that uses a self-consistent electric field description, calculating the electric potentials from a Poisson equation with RAM-generated FACs as sources and sinks and a specified (but dynamic) ionospheric conductance model [Ridley et al., 2001]. This conductance model has sources from sunlight on the dayside, starlight (applied everywhere), and a smooth auroral oval of high conductance. Figure 2 shows an example Pedersen conductance plot, annotated to illustrate the various ways the conductance is altered in the different simulations.

[17] Several recent inner magnetospheric modeling studies with self-consistent electric field descriptions have used other auroral conductance models. Most notably is the Hardy et al. [1987] empirical model, used in studies such as Fok et al. [2001] and Sazykin et al. [2002]. Khazanov et al. [2003] used a more sophisticated approach, self-consistently calculating the conductance from both precipitating electrons and ions using the Galand and Richmond [2001] formulas. In the present study, the auroral conductance model is an analytical formula with its location and intensity defined the RAM-generated region 2 currents. This simplicity is intentional and helps to isolate the influences on inner magnetospheric dynamics from varying a single conductance parameter.

[18] There are five ways in which the conductance is changed between the RAM simulations performed for this study. The first is the amplitude of the oval conductance peak, which varies in time according to the relation used by Ridley et al. [2004]. Because those formulas were developed for region 1 current intensities, a scaling factor was included to account for the known offset in magnitude between region 1 and region 2 currents [e.g., Iijima and Potemra, 1976; Weimer, 1999]. This multiplier is a parameter that is varied in this study, and the results are discussed in section 4.1 below. The nominal setting is a multiplier of 5 , with a range of 1 to 10 used in other simulations.

[19] A second parameter of the conductance model is the latitudinal location of the oval peak relative to the RAM-generated FAC peak. Because the oval is a product of both region 1 and region 2 currents, and RAM is only calculating the latter, the oval peak should be shifted poleward of the calculated FAC peak. This oval shift is a free parameter of this study, and the results are discussed in section 4.2 below. The nominal setting is a shift of $5^{\circ}$, with shifts of $0^{\circ}$ to $10^{\circ}$ used in other simulations.

[20] A third parameter is the dawn-to-dusk tilt of the auroral oval. It is known that precipitating electrons are the major contributor to this conductance, and their drift paths bring them closer to the Earth on the dawnside than on the duskside of the inner magnetosphere. Therefore, while holding the duskside poleward shift at $5^{\circ}$, the dawnside location of the oval is tilted equatorward a bit. The results of these simulations are discussed in section 4.3. The nominal tilt setting is $0^{\circ}$, with other simulations using tilts of up to $5^{\circ}$.

[21] The fourth parameter that this study is examining is the choice of the potential description applied at the highlatitude boundary of the Poisson equation $\left(72^{\circ}\right.$ magnetic latitude). Two models are used for this boundary condition: a ring of potential values extracted from the Weimer-96 model (the nominal case), and a sine wave description using only the cross polar cap potential difference from the Weimer-96 model. This latter case resembles a VollandStern two-cell convection pattern [Volland, 1973; Stern, 1975]. The results of this comparison are discussed in section 4.4. The nominal setting uses the Weimer-96 boundary condition.

[22] The final free parameter that is varied in this study is the baseline conductance value, to so-called starlight Pedersen conductance [Strobel et al., 1974]. The baseline (that is, nonauroral) ionospheric conductance at any given place on the nightside is not well known, but it is a function of the illumination from stars and scattered sunlight, from the length of time that part of the atmosphere has been in darkness, transport effects, plasmaspheric downflow and transport effects, and local heating influences. Therefore a wide range of baseline conductances will be used in this study to quantify the effect of this setting. The results of changing this value are presented in section 4.5. The nominal setting is a $\Sigma_{\mathrm{P}}$ of $1.0 \mathrm{~S}$, with other simulations using values from $0.1 \mathrm{~S}$ to $5.0 \mathrm{~S}$.

[23] For each simulation, the setting for each of these five parameters is held constant in time. In reality, all five of these parameters could be (and probably are) time dependent, and in fact they could be dependent on each other. No attempt is made here to determine a best-fit time series for the conductance parameters.

\section{Results}

[24] This study has two purposes. The first objective is to conduct a parametric study of the response in the numerical simulation results to various settings for the nightside ionospheric conductance. The second objective is to compare these results against observations to determine whether (and why) these systematic changes in the conductance bring the simulation results closer to reality (or not).

[25] For this second task, three different data sets will be employed. One data set will quantify the total energy content on the hot ions in the inner magnetosphere, yielding an assessment of the bulk inflow and outflow of particles and energy into the ring current. The other two data sets will test the resulting electric potential patterns by comparing against the spatial morphologies for two particular ion energy ranges. One population to be considered is the plasmasphere, consisting of cold ions of near-zero energy. These particles are greatly affected by the inner magnetospheric electric field, including a "memory" of the timehistory of the electric field (because the particles take many 

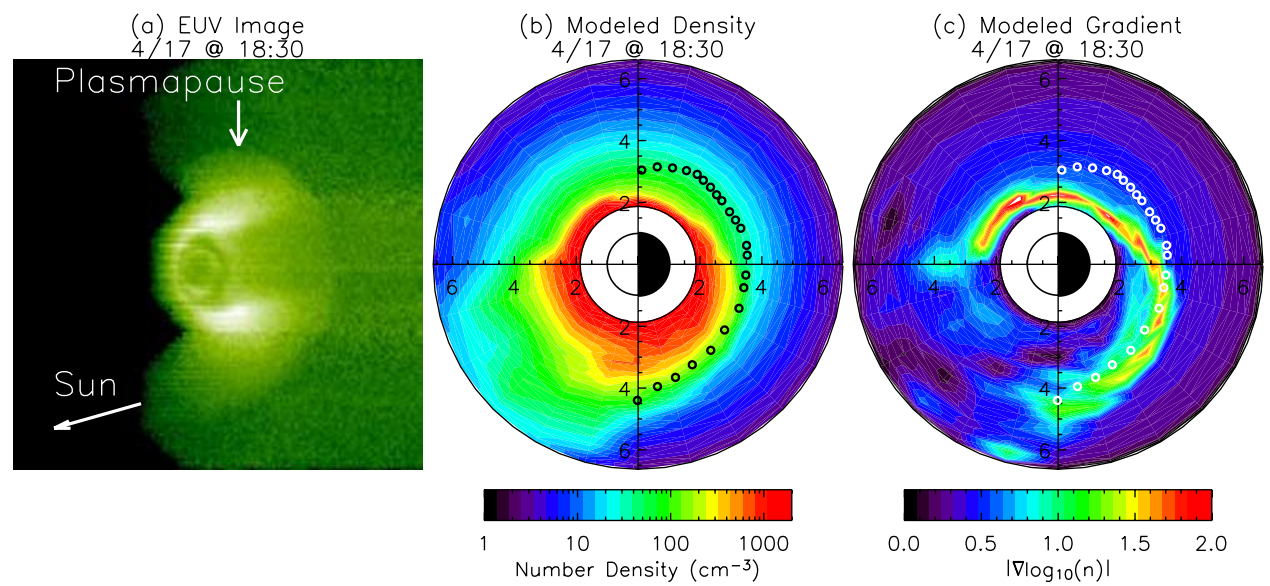

Figure 3. Example data-model comparison (at 1830 UT) between an IMAGE EUV snapshot and a DGCPM plasmasphere result. Shown here is (a) an EUV image (count rates per pixel) on a linear greenshade color scale, (b) DGCPM equatorial plane cold plasma densities on a logarithmic color scale, and (c) the magnitude of the gradient of the logarithm of the density from DGCPM on a linear color scale. In the EUV image, the view is over the North Pole with the Sun off to the left and slightly downward, as indicated. The plasmapause is also indicated on the image. In the model results, the view is over the North Pole with the Sun directly to the left, and distances are given in $R_{E}$. The circles (black in Figure $3 \mathrm{~b}$ and white in Figure $3 \mathrm{c}$ ) are plasmapause locations extracted from the EUV image.

hours to convect through near-Earth space). The other energy range to be considered is one around the peak of the storm-time ring current distribution; that is, around $50 \mathrm{keV}$.

[26] The first data set is the $D s t^{*}$ time series for the second half of 17 April. The Dessler-Parker-Sckopke (DPS) relation [Dessler and Parker, 1959; Sckopke, 1966] is used to convert the total energy content of the hot ions within the simulation domain into a magnetic perturbation value at the center of the Earth $\left(\Delta B_{\text {DPS }}\right)$. While there are many known caveats to using the DPS relation in direct comparison against Dst* [e.g., Carovillano and Siscoe, 1973; Turner et al., 2000; Liemohn, 2003], it is believed that comparing $\Delta B_{\text {DPS }}$ with $D s t^{*}$ is a crude but acceptable method of quantifying the overall energy content of the ring current [e.g., Greenspan and Hamilton, 2000].

[27] The second data set to be used in this study is that from the extreme ultraviolet (EUV) instrument [Sandel et al., 2000] on the IMAGE satellite [Burch, 2000, 2003], which counts $30.4 \mathrm{~nm}$ photons. The main magnetospheric source of this light is photons scattered by plasmaspheric $\mathrm{He}^{+}$. The line-of-sight flux integrals can be inverted (crudely or rigorously) to yield maps of the cold plasma density in the equatorial plane. The plasmapause [Carpenter, 1963] is usually evident in these images [e.g., Sandel et al., 2003]. The motion of the plasmapause can be analyzed to extract the convection electric field. Such studies have been conducted with semiempirical convection models [e.g., Goldstein et al., 2002], by simple analytical convection models [e.g., Goldstein et al., 2003], and by more sophisticated convection models [e.g., Liemohn et al., 2004]. Electric fields have also been directly determined from the EUV data [Goldstein et al., 2004, 2005].

[28] IMAGE was in a nearly noon-midnight orbit (always within $1.5 R_{E}$ of the $Y_{\mathrm{SM}}=0$ axis), with apogee a bit westward of midnight about $45^{\circ}$ above the equatorial plane. IMAGE ascended across the SM equatorial plane around
1100 UT on 17 April and reached apogee around 1700 UT. Around 2145 UT, IMAGE made its closest approach to the $+Z_{\mathrm{SM}}$ axis for this orbit (roughly $5 R_{E}$ geocentric distance above the North Pole), and then IMAGE rapidly swung close to Earth on its perigee pass (see Figure 5 of Liemohn et al. [2004] for a plot of this IMAGE orbit). Data from IMAGE can therefore be used from roughly 1600 UT until 2230 UT, an interval including the late main phase and most of the recovery phase of the magnetic storm.

[29] Figure 3 shows a qualitative data-model comparison between an EUV image and a DGCPM plasmasphere result. Figure $3 \mathrm{a}$ presents an EUV snapshot at 1830 UT on 17 April, a time close to the $D s t^{*}$ minimum. IMAGE is located at $\left(4.5 R_{E}, 0,6.5 R_{E}\right)$ in the $\mathrm{SM}$ coordinate frame. The Sun is to the left and slightly downward, as indicated on the plot. Note that much of the dayside is blocked out by a voltage ramp-down to prevent direct sunlight from entering the camera. The plasmapause is seen in this image as the sharp color change a few $R_{E}$ from Earth, as indicated in Figure $3 \mathrm{a}$. Figure $3 \mathrm{~b}$ shows a DGCPM cold plasma density result for 1830 UT from the simulation with nominal conductance settings (as described in section 3). The EUV plasmapause values that were manually extracted from Figure $3 \mathrm{a}$ are drawn on Figure $3 \mathrm{~b}$ as small black circles. Liemohn et al. [2004] argued that observed plasmapause locations are best compared against the maximum gradient in $\log$ (density) rather than against the absolute value of the density, and this method will be followed in the present study. Therefore Figure 3c shows a comparison of $\left|\nabla \log _{10}(n)\right|$, where $\mathrm{n}$ is the DGCPM density shown in Figure $3 \mathrm{~b}$, against the observed plasmapause locations (white circles in this plot). This is the data-model comparison to be conducted for the EUV images.

[30] The third data set to be used for this analysis is that from the IMAGE high-energy neutral atom (HENA) imager [Mitchell et al., 2000]. It detects hot atoms in the energy range from $\sim 10 \mathrm{keV}$ to $>200 \mathrm{keV}$, covering the bulk of the 

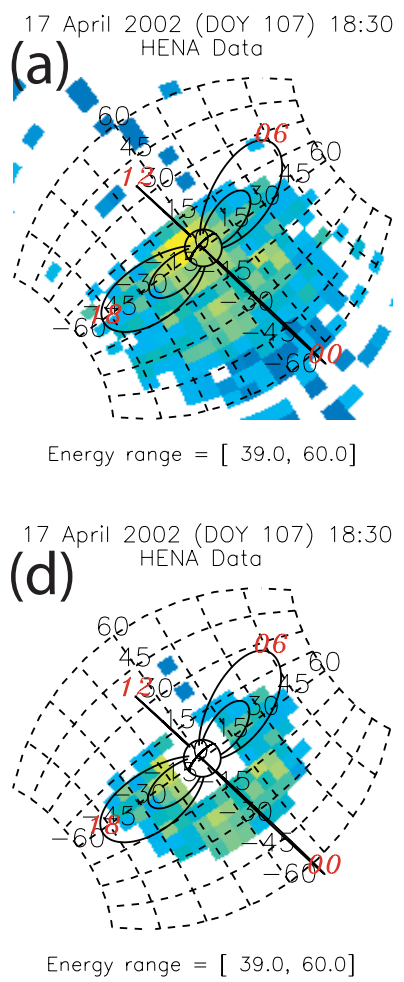

17 April 2002 (DOY 107) 18:30

(b) Simulation Results
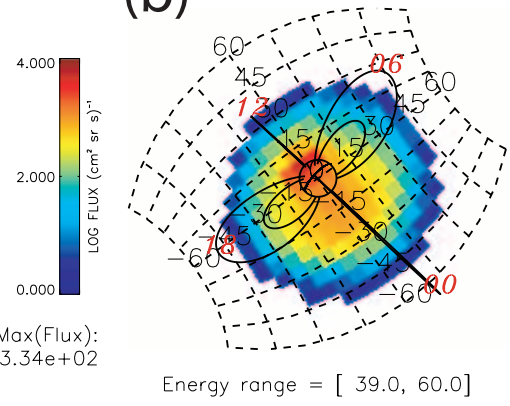

17 April 2002 (DOY 107) 18:30

(e)

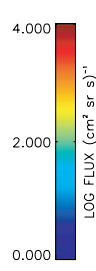

$\operatorname{Max}($ Flux): Max(Flux):
$2.01 \mathrm{e}+02$

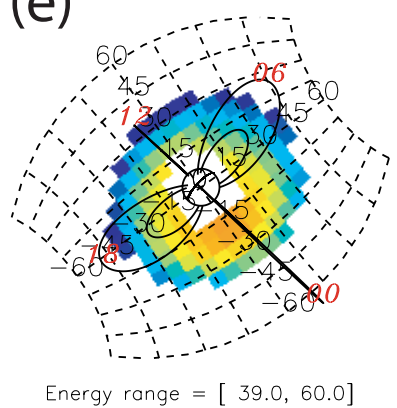

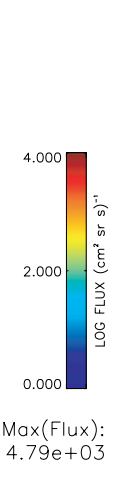

Ring Current Simulation Results Energy range $=[390600]$
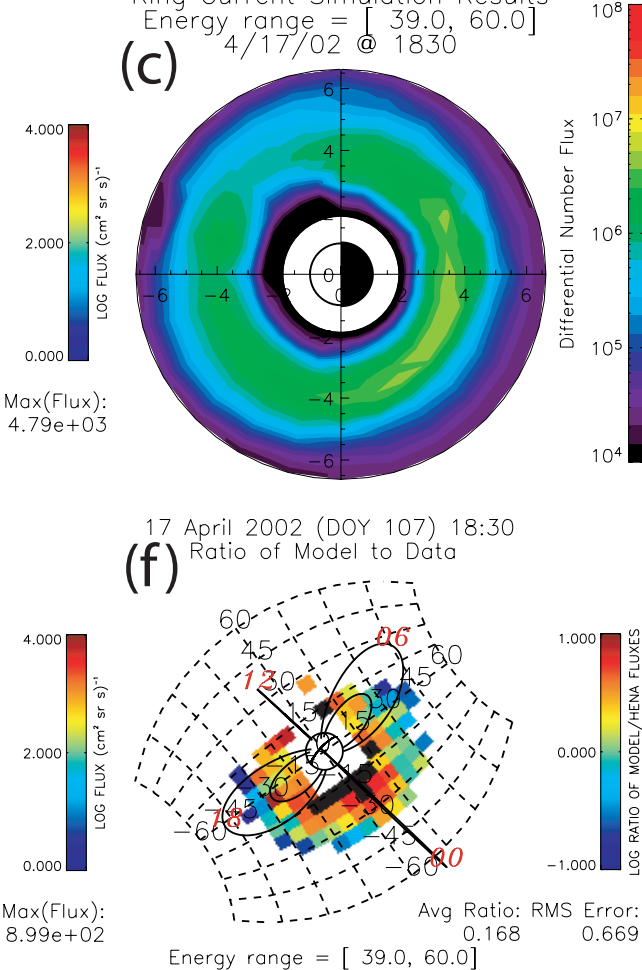

17 April 2002 (DOY 107) 18:30

(f)

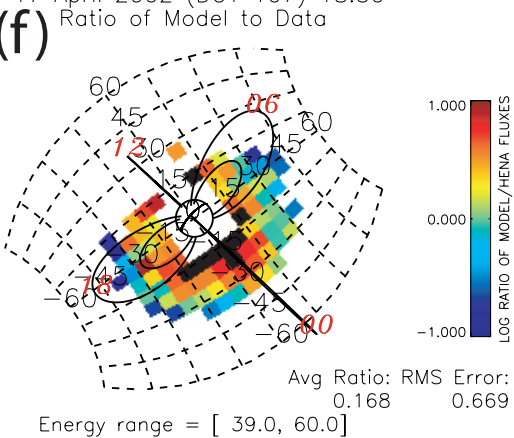

Figure 4. Example data-model comparison (at 1830 UT) between an IMAGE HENA snapshot and a RAM hot ion result (39-60 keV energy range). Shown here is (a) HENA image (particle flux integrated over the energy channel) on a logarithmic color scale, in units of $\mathrm{cm}^{-2} \mathrm{~s}^{-1} \mathrm{sr}^{-1}$, (b) simulated image from the RAM results in an equivalent format, (c) equatorial plane hot ion number fluxes from RAM averaged over the selected energy channel on a logarithmic color scale, in units of $\mathrm{keV}^{-1} \mathrm{~cm}^{-2} \mathrm{~s}^{-1} \mathrm{sr}^{-1}$, (d) HENA image showing only the pixels in an annulus of $2-5.5 R_{E}$ equatorial plane radial distances, (e) simulated image from RAM showing only the pixels in the $2-5.5 R_{E}$ annulus, and (f) the ratio of the simulated image to the observed image in the $2-5.5 R_{E}$ annulus on a logarithmic color scale. The view in the ENA images is from SM coordinates $(-4.5,-0.1,6.5)$ in $R_{E}$, along the midnight meridian $55^{\circ}$ above the equatorial plane. Dipole field lines at $L=4$ and 8 are drawn on the plots for reference, and the local times are listed in red. For the RAM equatorial plane plot, the view is over the North Pole with the Sun to the left, and distances are given in $R_{E}$.

ring current as well as the tail of the plasma sheet energy range. It has been used to examine the plasma morphology during both substorms [e.g., Brandt et al., 2002a, 2002b] and storms [e.g., Mitchell et al., 2001, 2003; Brandt et al., 2002c, 2002d]. Comparisons with in situ data are quite reasonable [Vallat et al., 2004], and data-model comparisons have also proved fruitful [e.g., Fok et al., 2003; Ebihara and Fok, 2004].

[31] Figure 4 is an example data-model comparison between a HENA image and a RAM simulation result. This chosen time, 1830 UT, was selected on the basis of the HENA data. There was a ring current enhancement at around 1600 UT and there was a clear substorm injection at around 1904 UT (as seen by FUV/WIC and HENA). The time around 1830 was a relatively steady period for the ring current with a moderate partial ring current on the nightside. Figure 4a shows the HENA data for 1830 UT on 17 April for the 39-60 keV energy channel. Note that the displayed fluxes are integrated (not averaged) over this range. The dayside portion of the image is blocked out by a shutter to avoid direct sunlight entering the instrument. Figure $4 \mathrm{~b}$ shows a simulated HENA image, created by forward modeling the RAM simulation results from the run with nominal conductance settings. That is, line-of-sight integrations of ENA production were computed through the RAM results with the same grid resolution as the HENA instrument, using the algorithms and codes described by DeMajistre et al. [2004] (see equation (2) of that paper for the equation relating ENA flux to ion flux). Note that the forward modeling routine used for this procedure (as supplied by the HENA team) assumes isotropy of the hot ion pitch angle distribution at all locations. The main error that this assumption introduces is a very bright signal close to the planet because the loss cone is assumed filled in the ENA forward modeling procedure when in reality (and in RAM) it is not. Figure 4c shows the equatorial plane $\mathrm{H}^{+}$flux distribution used to create the simulated image in Figure 4b. To make a worthwhile comparison between the HENA image and the simulated image, it is useful to only consider those parts of the image where both data sets are valid. So, Figure 4d (Figure 4e) shows a "masked" version of Figure 4a (Figure 4b), only including those data points with lines-of-sight that cross the magnetic equatorial plane between $L=2$ and $L=5.5$. The 
point-spread function of the HENA instrument in this energy range is about $12 \times 8$ (elevation $\times$ azimuth) degrees, which is smaller than the masked region. This means that the point-like low-altitude ENA emissions did not spread outside the masked region. The log of the ratio of the simulated fluxes to the observed values, using only pixels where there is valid data in both Figures $4 \mathrm{~d}$ and $4 \mathrm{e}$, is presented in Figure 4f. A "green" value of zero therefore means the observed and modeled ENA fluxes match. The black pixels near Earth are values that exceed 1.0. It is this final image that will be the basis of the datamodel comparisons between HENA and RAM.

[32] As stated in section 3, many simulations were performed, each with a different assumption about the nightside ionospheric conductance (or the high-latitude boundary condition). The results for each of the parameters will be discussed in the following subsections, but first let us present an example snapshot at 1830 UT data-model comparison for all of the simulations. Figure 5 shows EUV-DGCPM plasmapause comparisons for all 16 of the simulations conducted for this study (at 1830 UT on 17 April). Each dial plot is similar to Figure 3c, and in fact the results for the nominal conductance setting are repeated down the center column. That is, Figures $5 \mathrm{c}, 5 \mathrm{~h}$, $5 \mathrm{k}, 5 \mathrm{n}$, and $5 \mathrm{r}$ are all identical, using a conductance multiplier of 5 , a $5^{\circ}$ poleward shift of the oval, a dawnside equatorward tilt of $0^{\circ}$, a Weimer high-latitude boundary condition, and a baseline Pedersen conductance of 1.0 S. Each row show results for the simulations varying a particular parameter (and holding the others fixed), and the description of what is shown in each row is given in the caption. The details of these plots will be examined below; they are introduced here so that the data-model comparisons for all of the simulations can be seen in one place and directly compared. These plots also provide a qualitative, graphical interpretation of the quantitative line plots presented later.

[33] Analogously, Figure 6 shows HENA-RAM comparisons for all 16 of the simulations at 1830 UT on 17 April. Each dial plot is similar to Figure 4f, and the center column (being the nominal conductance setting simulation) is identical to that plot. As with Figure 5, these plots will be discussed in detail in the subsections below, and are presented here to provide a means of directly comparing all 16 simulations against each other.

[34] A final plot to be introduced here is Figure 7, which shows inner magnetospheric electric potential patterns for the 16 simulations at 1830 UT on 17 April. Equipotential contours are drawn every $8 \mathrm{kV}$. As with Figure 5 and 6 , the details of these plots will be discussed later and are shown here as an example of the electric potential structures created from each choice of the ionospheric conductance.

\subsection{FAC- $\Sigma$ Strength Relationship}

[35] Figure 8 shows a summary of the data-model comparisons for the simulations with various conductance intensities. This is the first of the five conductance model parameters discussed in section 3 above. The adjustable parameter is the multiplication factor relating the peak FAC intensity to the peak auroral conductance value. The three lines in each panel are the results for three of the simulations (multiplier of $M=1,5$, and 10 for the solid, dotted, and dashed lines, respectively). Figure 8a shows a comparison of the observed $D s t^{*}$ with $\Delta B_{\text {DPS }}$. It is seen that the $M=1$ result produces too little of a disturbance, while the $M=10$ result has a consistently deeper magnetic depression than the measured time series. The $M=5$ case (the nominal conductance settings) appears to be the closest to the observed $D s t^{*}$

[36] Figures $8 \mathrm{~b}$ and $8 \mathrm{c}$ show quantitative comparisons between the EUV plasmapause extractions and the DGCPM results for these three simulations. For each UT, the observed $L$ value of the plasmapause was compared against the modeled $L$ value at that MLT, and then the values were averaged over all of the data points. Figure $8 \mathrm{~b}$ shows the ratio of the modeled $L$ value to the observed $L$ value minus one, while Figure $8 \mathrm{c}$ shows the root-meansquare error of this value. For an accurate simulation therefore, the lines in Figures $8 \mathrm{~b}$ and $8 \mathrm{c}$ should be close to zero. It is seen that the $M=1$ conductance produced a consistently larger plasmasphere than the observed one, while the $M=10$ conductance yielded a smaller plasmasphere than observed. Note that the IMAGE orbit for 17 April is such that, during the early portion of the orbit, only the nightside is being observed, and the dayside plasmasphere is not fully seen until after 2000 UT. Figure $8 \mathrm{c}$ reveals that all three simulations have roughly equal RMS errors for the plasmapause, indicating good agreement on the nightside. The $M=1$ result worsens late in the orbit, indicating a poor comparison on the dayside for this simulation.

[37] Figures $5 \mathrm{a}-5 \mathrm{e}$ illustrate the spatial comparison behind the line plots of Figure $8 \mathrm{~b}$ and $8 \mathrm{e}$. It is clear that the modeled plasmapause moves inward with increasing multiplier value. All of the simulations, however, predict an indentation of the plasmapause location in the predawn sector (upper right quadrant of each dial plot) that is not seen in the data. The reason for this indentation is given in Figure 7. Figures $7 \mathrm{a}-7 \mathrm{e}$ all show a convection electric field with a radially inward component in the predawn sector. In addition, the evening sector often shows a difference between the observed and modeled plasmapause location.

[38] Figures $8 \mathrm{~d}$ and $8 \mathrm{e}$ show the comparisons between the HENA images and the simulated images from the RAM results for the 39-60 keV energy channel. For each UT, all of the valid points within the spatial region of interest (either the ratio of the logarithm of the fluxes or the RMS error of this ratio) are averaged to produce a single value. Therefore the closer the values are to zero the better the agreement between the data and the model results. It is seen that there are two intervals with distinct comparison characteristics, with a transition between them at about 1930 UT. At earlier times, the simulated ENA fluxes are, on average, at or above the observed values. At later times, the simulated values are, on average, at or below the measurements. The main difference between these two intervals is the extension of the observations to the dayside as IMAGE progresses along its orbit track. In general, the results with the $M=1$ conductance setting were best during the main phase and early recovery phase, and the $M=5$ or 10 results were best during the late recovery phase (in both Figures 8d and 8e).

[39] To understand the drop in average RAM-to-HENA flux ratio during the storm, it is useful to examine a time 
(a) Mult $=1 \mathrm{x}$

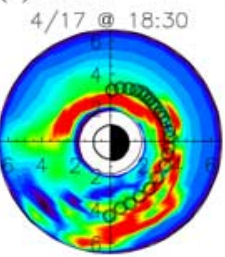

(f)

(f) $\mathrm{Shift}=0^{\circ}$
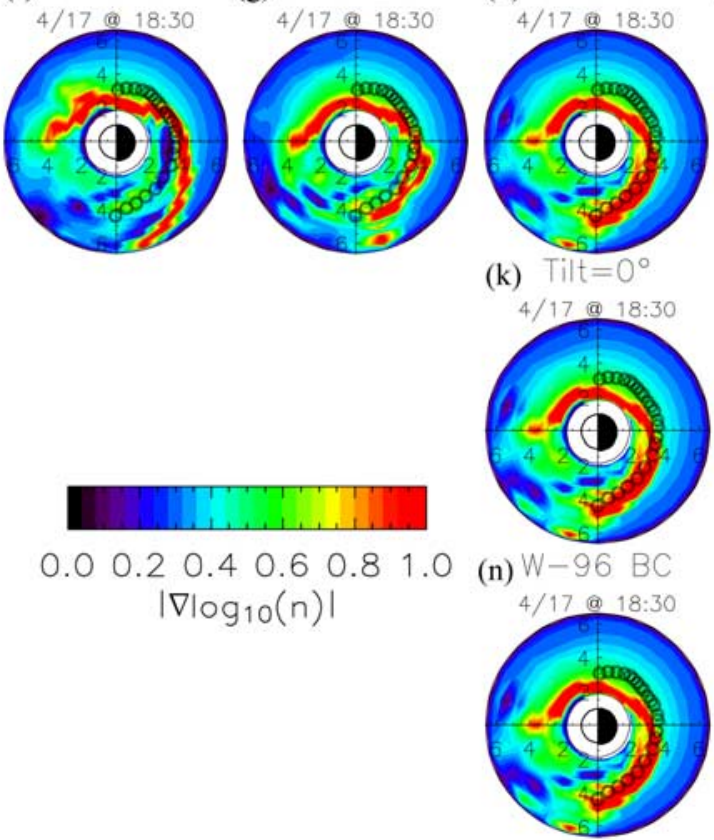

$(p)^{\Sigma p}=0.1 \mathrm{~S}$

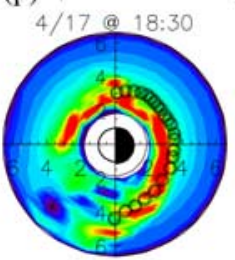

(q) $\sum p=0.5 \mathrm{~s}$

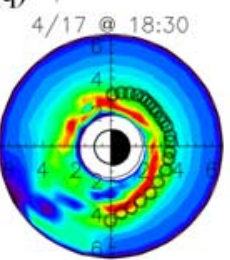

(g) Shift $=2.5^{\circ}$
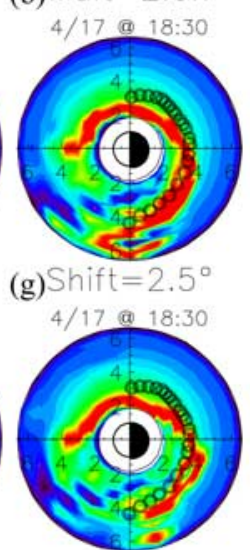

(k)

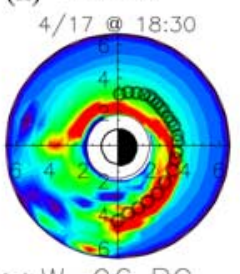

(n)

(c) Mult $=5 x$

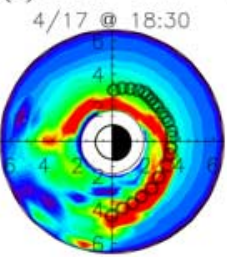

(h) Shift $=5^{\circ}$

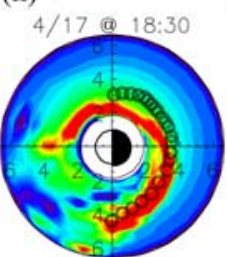

k) Tilt $=0^{\circ}$

$\left|\nabla \log _{10}(n)\right|$ (r) $\sum p=1.0 \mathrm{~s}$

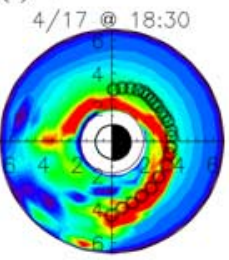

(d) Mult $=7.5 x$

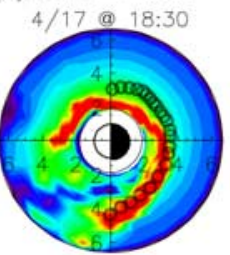

(i) Shift $=7.5^{\circ}$

(i)

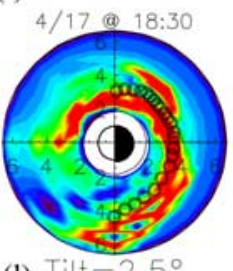

(1) $\mathrm{Tilt}=2.5^{\circ}$

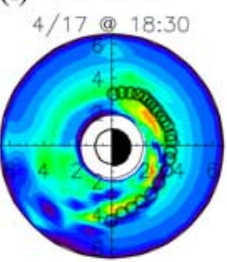

(o) $\mathrm{V}-\mathrm{S} B \mathrm{BC}$

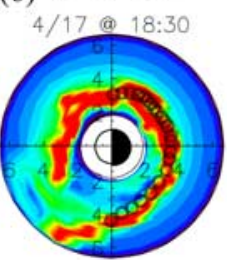

(s) $\sum p=2.5 \mathrm{~S}$ (e) Mult $=10 \mathrm{x}$

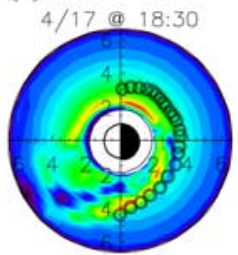

(j) Shift $=10^{\circ}$

4/17@ 18:30

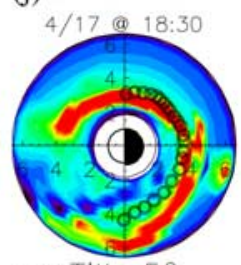

(m)

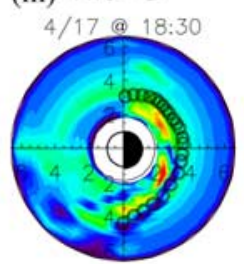

t) $\Sigma_{p}=5.0 \mathrm{~S}$
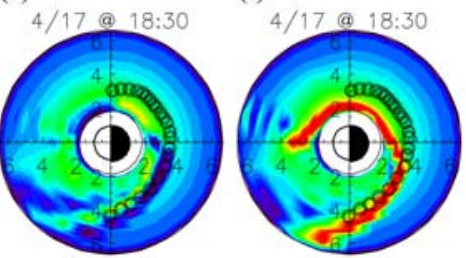

Figure 5. EUV-DGCPM plasmapause comparisons at 1830 UT. Each plot has the same format as Figure $3 \mathrm{c}$, and the five plots down the center column are the same data as in that plot (the case with the nominal settings for each parameter). Each row shows the results of varying a different parameter in the ionospheric conductance specification, and will be discussed in more detail throughout section 4 . The top row $(\mathrm{a}-\mathrm{e})$ shows the influence of varying the magnitude of the auroral oval conductance (relative to the field-aligned current magnitude). The second row ( $\mathrm{f}-\mathrm{j}$ ) shows the influence of varying the poleward latitudinal offset between the peak FAC value (from RAM) and the peak auroral oval conductance. The third row $(\mathrm{k}-\mathrm{m})$ shows the influence of stretching the dawnside location of the oval conductance equatorward. The fourth row $(n-0)$ shows the influence of the high latitude potential specification. The fifth row $(p-t)$ shows the influence of the baseline Pedersen conductance value (applied everywhere).

series of the observed and simulated ENA images. Figure 9 presents this comparison: the top two rows show simulated ENA plots for the $M=1$ and $M=10$ settings (respectively) at five times during the IMAGE orbit (space 1.5 hours apart) and the bottom row shows the corresponding HENA images. Note that the images have been truncated to only show the spatial region of interest, like in Figures 4d and 4e. In the first four HENA images (Figures $9 \mathrm{k}-9 \mathrm{n}$ ), there is a clear ENA peak in the premidnight sector. This peak is also seen in the corresponding simulated ENA images, with the $M=1$ results providing a better match to the observed intensity. The HENA image at 2200 UT, however (Figure 9o), is very different, with a flux peak near local noon. In the intervening HENA images (not shown), it is clear that the flux peak progressively shifts westward roughly 10 hours of local time in the 1.5 hours between 
(a) Mult $=1 \mathrm{x}$

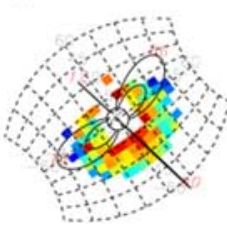

(b) Mult $=2.5 x$

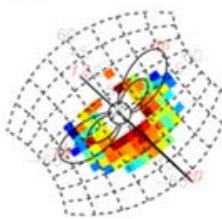

(f) Shift $=0^{\circ}$

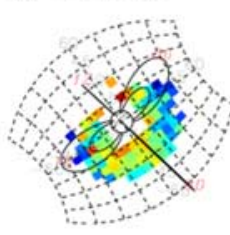

(g) Shift $=2.5^{\circ}$
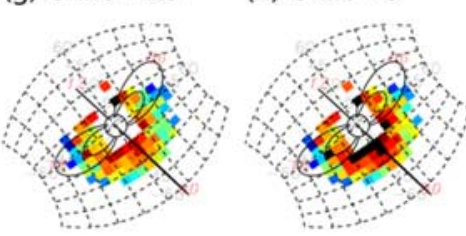

(k) Tilt $=0^{\circ}$
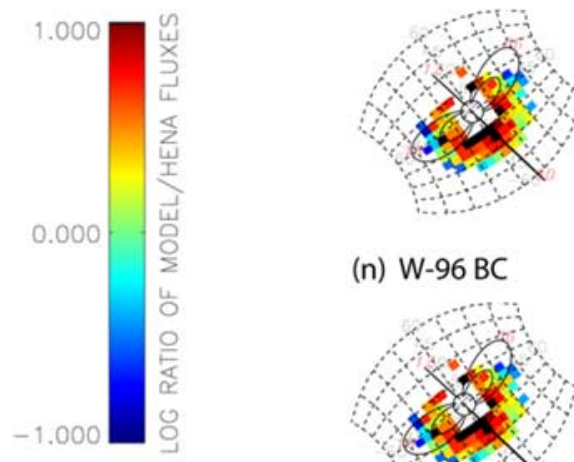

(n) $W-96 B C$

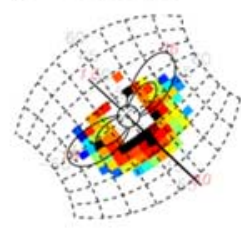

(p) $\Sigma_{p}=0.1 \mathrm{~S}$

(q) $\Sigma_{p}=0.5 \mathrm{~S}$
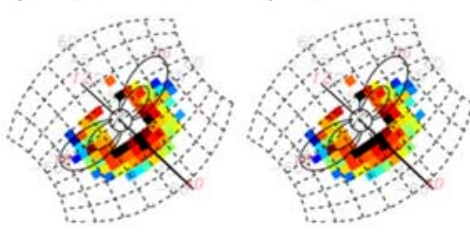

(c) Mult $=5 \mathrm{x}$
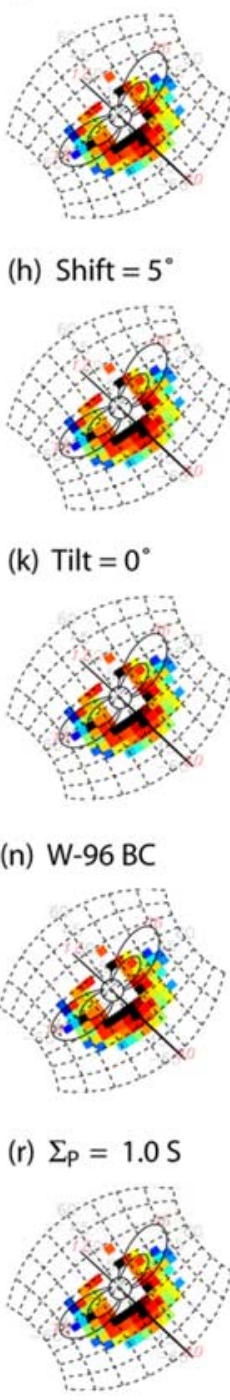

(d) Mult $=7.5 \mathrm{x}$

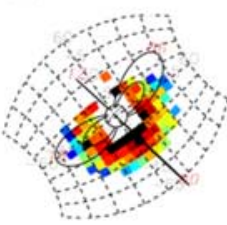

(e) Mult $=10 x$

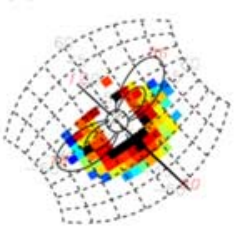

(i) Shift $=7.5^{\circ}$

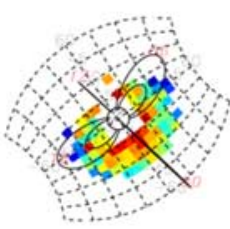

(j) Shift $=10^{\circ}$

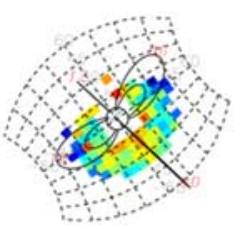

(I) Tilt $=2.5^{\circ}$

(m) Tilt $=5^{\circ}$
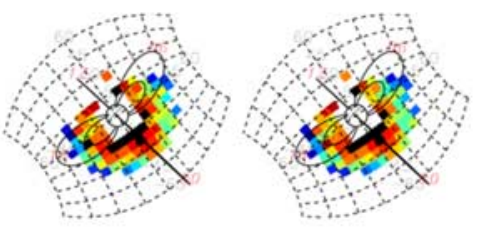

(o) V-S BC

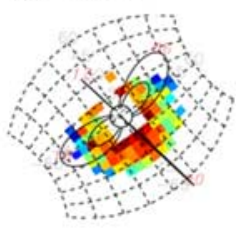

(s) $\Sigma_{p}=2.5 \mathrm{~S}$

(t) $\Sigma_{p}=5.0 \mathrm{~S}$
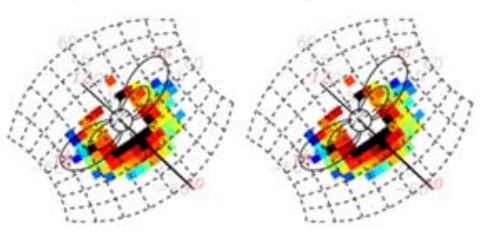

Figure 6. RAM-to-HENA image ratios at $1830 \mathrm{UT}$ for the $2-5.5 R_{E}$ equatorial plane annulus. Each plot has the same format as Figure 4f, and the five plots down the center column are reproductions of that plot. The five rows are the same as in Figure 5.

the images in Figures 9n and 9o. The RAM-generated ENA images, however, do not show this MLT shift in the location of the flux peak. The reason for this discrepancy is unclear and will be addressed in section 5 below.

[40] A clear feature of Figure 9 is that the addition of the dayside into this data-model comparison introduces a ring of low ENA flux pixels around the outside of the spatial region of interest. These pixels bring down the model-todata flux ratio plotted in Figure 8d.

[41] Table 1 lists the average values (ratio and RMS error) for each of the data-model comparisons (averaged over UT): $D s t^{*}$ versus $\Delta B_{\mathrm{DPS}}$; EUV versus DGCPM plasmapause $L$ value; and HENA versus RAM 39-60 keV $\log$ (flux) of energetic neutral atoms in our defined region of interest. The final number in each row is the "total" error for that simulation relative to the chosen data, calculated as an RMS error of the six other error values listed in the table (equally weighted). The best number for each column (that is, the value closest to zero) is highlighted in bold. The FAC-to- $\Sigma$ relationship portion of Table 1 shows that the $M=5$ simulation was the best of this set of runs at reproducing the data (taken altogether). Of course, this is a huge contraction of the data-model comparisons into a single number for each simulation. For five of the six other columns, however, the error estimates either increase or decrease monotonically with $M$. The $M=5$ results are the best in only two of the six parameters. Note that the $M=1$ simulation had the best value in the HENA-RAM RMS error column (for all 16 simulations).

\subsection{Poleward Offset of the Oval}

[42] Figure 10 shows the data-model comparisons for the simulations with various poleward offsets of the auroral conductance (relative to latitude of the RAM-generated 
(a) Mult $=1 \mathrm{x}$

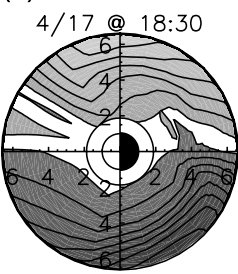

(f) Shift $=0^{\circ}$

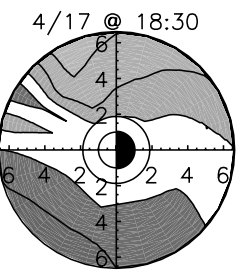

(g) Shift $=2.5^{\circ}$

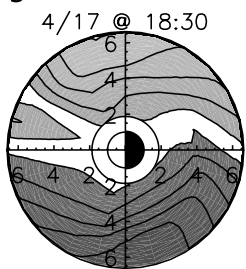

(c) Mult $=5 x$

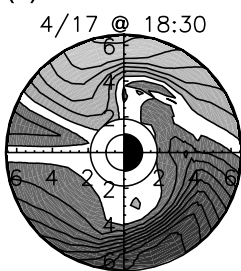

(h) Shift $=5^{\circ}$

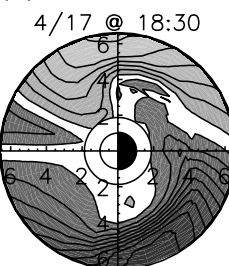

(k) Tilt $=0^{\circ}$

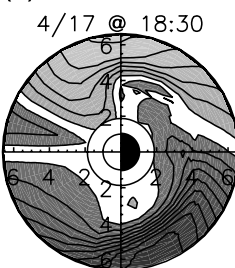

(n) $\mathrm{W}-96 \mathrm{BC}$

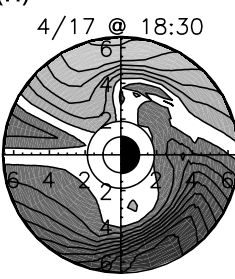

(r) $\Sigma_{P}=1.0 \mathrm{~S}$

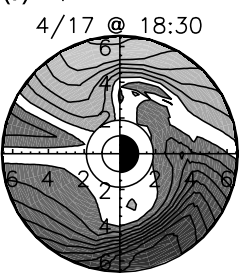

(d) Mult $=7.5 x$

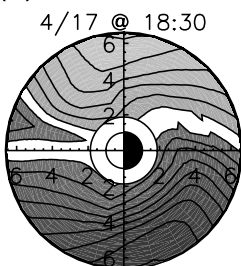

(i) Shift $=7.5^{\circ}$

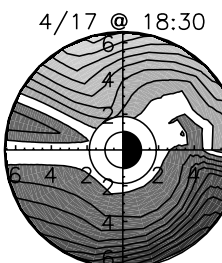

(I) Tilt $=2.5^{\circ}$

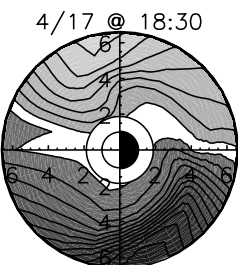

(o) $\mathrm{V}-\mathrm{S} B \mathrm{BC}$

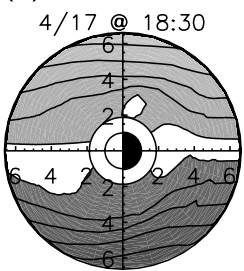

(s) $\Sigma_{P}=2.5 \mathrm{~S}$

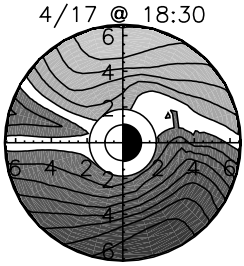

(e) Mult $=10 x$

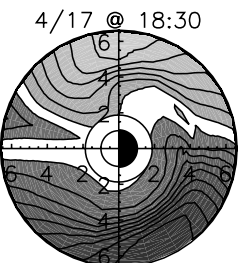

(j) Shift $=10^{\circ}$

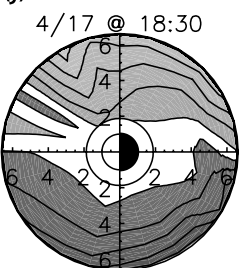

(m) Tilt $=5^{\circ}$

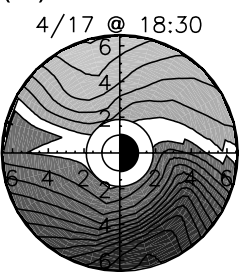

(t) $\Sigma_{P}=5.0 \mathrm{~S}$

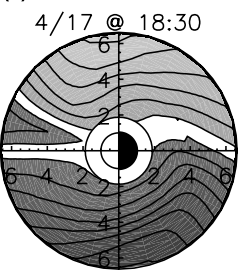

Figure 7. Electric potential contours in the inner magnetosphere at 1830 UT for the 20 simulations shown in Figures 5 and 6. Equipotentials are drawn every $8 \mathrm{kV}$.

FAC peak). The format is the same as Figure 8, except that the three lines show a poleward shift of $S=0^{\circ}, 5^{\circ}$, and $10^{\circ}$ (solid, dotted, and dashed, respectively).

[43] There are many similarities between Figures 8 and 10. For instance, all three runs reproduce the general shape of the $D s t^{*}$ perturbation for this storm, although the magnitudes are different between the three simulations. Another similar feature is the upward trend of the plasmapause RMS error late in the day. A third similarity between Figures 8 and 10 is the decrease in the $39-60 \mathrm{keV}$ ENA flux ratio around $1900 \mathrm{UT}$.

[44] Some of the results in Figure 10 are not intuitive. The $S=5^{\circ}$ simulation is markedly better than the other two, even though its poleward shift is in between the other two simulations. This is also seen in the poleward shift portion of Table 1. None of the trends versus $S$ are monotonic (like they are against $M$ ) but rather reach a relative maximum or minimum value at (or near) $S=5^{\circ}$. The $S=5^{\circ}$ values in Table 1 are closest to zero in 4 of the 6 categories and nearly best overall. The $S=2.5^{\circ}$ simulation is very slightly better, as seen in the final column. This $S=2.5^{\circ}$ total error value is tied (with two other settings) for the best among all 16 simulations. The $S=2.5^{\circ}$ results are also tied for best in the $39-60 \mathrm{keV}$ ENA flux ratio column.

[45] The reason for this nonmonotonic influence of the oval shift setting is illustrated in the potential patterns of Figures $7 f-7 j$. It is seen there that the nightside electric fields are strongest for the $S=5^{\circ}$ simulation and are weaker for both larger and smaller poleward shift settings. Hot ions from the near-Earth plasma sheet are brought in faster and deeper by the stronger electric fields, thus creating a stronger (and, apparently, more realistic) storm-time ring current. 


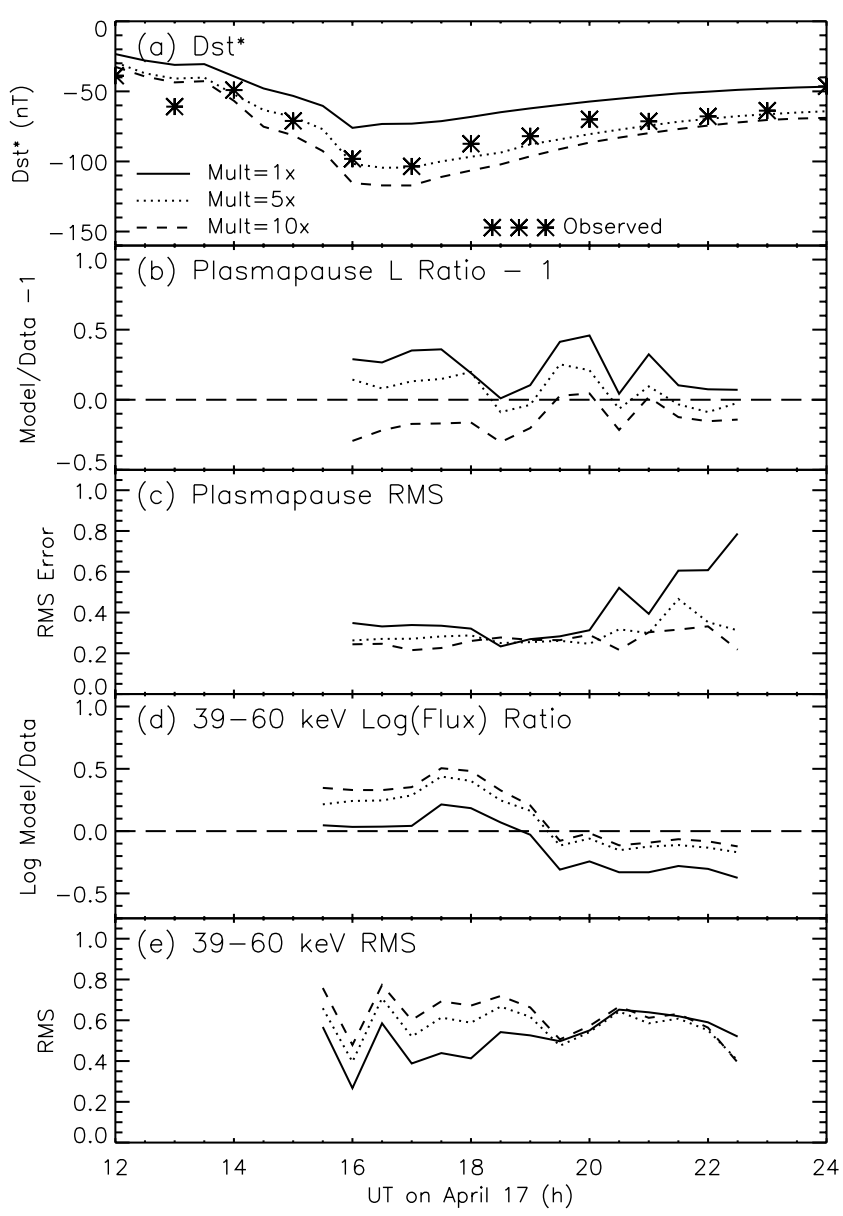

Figure 8. Quantitative data-model comparisons for the simulations with different FAC-to- $\Sigma$ magnitude relationships. The three lines in each plot show the results for a multiplier of 1 (solid line), 5 (dotted line), and 10 (dashed line). Shown in each plot is (a) observed $D s t^{*}$ (asterisks) versus simulated $D s t^{*}$ using the DPS relation, (b) the ratio of the simulated plasmapause radial distance location to the observed value (averaged over all MLT for each UT) minus one, (c) the RMS error in the simulated radial distance location compared to the observed value (averaged over all MLT for each UT), (d) the ratio of the log-flux values for the 39-60 keV energy range between the simulated ENA image and the observed values in the $2-5.5 R_{E}$ annulus (averaged over all nonzero pixels in the annulus at each UT), and (e) the RMS error in the log-flux ratios for the 39$60 \mathrm{keV}$ energy range (average over all nonzero pixels in the annulus at each UT). Horizontal long-dashed lines at zero are drawn in Figures $8 \mathrm{~b}$ and $8 \mathrm{~d}$ for reference.

[46] To explain this, consider Ohm's law. In its scalar form, Ohm's law is $j=\sigma E$, where $j$ is current, $\sigma$ is conductivity, and $E$ is electric field. The value of $j$ flowing horizontally in the ionosphere is dictated by the source-sink terms; that is, the partial ring current-generated FACs. When the oval of high conductance is coincident with these FACs $\left(S=0^{\circ}\right)$, the resulting electric fields in the inner magnetosphere are small. Because the oval of high conductance is shifted so far equatorward, there is a region of low conductance between the poleward edge of the auroral oval and the boundary of the Poisson equation solution. The potential specified at the Poisson equation boundary latitude is therefore "consumed" in this low conductance region, and only a small amount of electric potential drop spans the inner magnetosphere. What potential does exist there is undershielded due to the high conductance, and so plasma is convected into and through the simulation domain by the large-scale convection field. There is little accumulation within the inner magnetosphere, though, because the ions are swept out the dayside as fast as they are swept in the nightside.

[47] When the oval of high conductance does not overlap with these FACs $\left(S=10^{\circ}\right)$, then the shielding electric fields are large. After an initial injection of plasma, the large electric fields not only attempt to destroy the pressure peak but also efficiently prevent additional injections. This results in small-scale structure in the plasma pressure distribution and a smaller total energy content of the hot ions.

[48] Figure 11 provides a pictorial illustration of these features in the pressure distributions. Shown are the total hot ion pressure $\left(\mathrm{H}^{+}\right.$and $\mathrm{O}^{+}$combined for all particle energies calculated in the simulation) in the equatorial plane for the simulations with $S=0^{\circ}, 5^{\circ}$, and $10^{\circ}$ oval shifts at two times during the storm (1600 and 1900 UT). It is seen that the pressures from the $\mathrm{S}=0^{\circ}$ setting (Figures 11a and 11b) are smoother than those from the other simulations. It is also seen that there is substantial structure in the pressure distributions (and the pressures are smaller) of Figures $11 \mathrm{c}$ and $11 \mathrm{f}$ (relative to the other simulations).

[49] The significant amount of small-scale structure in the $\mathrm{S}=5^{\circ}$ results (Figures $11 \mathrm{~b}$ and $11 \mathrm{e}$ and also seen in Figure $7 \mathrm{~h}$ ) is evidence of the multiple injections into the region. With each injection, a potential well-peak pair is formed, with a potential minimum colocated with the upward FACs on the eastern half of the pressure peak and a potential maximum colocated with the downward FACs on the western half of the pressure peak. Liemohn and Brandt [2005] discuss the formation of these "vortex pairs" in detail. One finding of that study is that the smallscale structure in the plasma distribution is not resolved in the HENA data. The magnitude and morphology of the potential peaks depend on many factors, especially the features of the plasma injection and the local ionospheric conductance. Therefore it is felt that these small-scale structures are geophysically real and not a numerical artifact, but a definitive resolution on their existence must wait for more conclusive observational evidence.

\subsection{Dawn-Dusk Tilt of the Oval}

[50] Figure 12 shows the data-model comparisons for the simulations with various dawnside-only shifts in the oval location. The three simulations shown on the plots all have a duskside poleward shift of $5^{\circ}$, with the dawnside magnetic latitude tilted equatorward by $T=0^{\circ}, 2.5^{\circ}$, and $5^{\circ}$ from this poleward shift (solid, dotted, and dashed lines, respectively). That is, a tilt setting of $5^{\circ}$ means the conductance peak at 0600 MLT is at the same latitude as the RAM-generated FAC peak.

[51] Figure 12 exhibits many of the same features as those seen in Figures 8 and 10. All three simulations do very well at reproducing the observed $D s t^{*}$, in both shape and 
$\begin{array}{lllll}\text { (a) } M=1 @ 16: 00 & \text { (b) } M=1 @ 17: 30 & \text { (c) } M=1 @ 19: 00 & \text { (d) } M=1 @ 20: 30 & \text { (e) } M=1 @ 22: 00\end{array}$
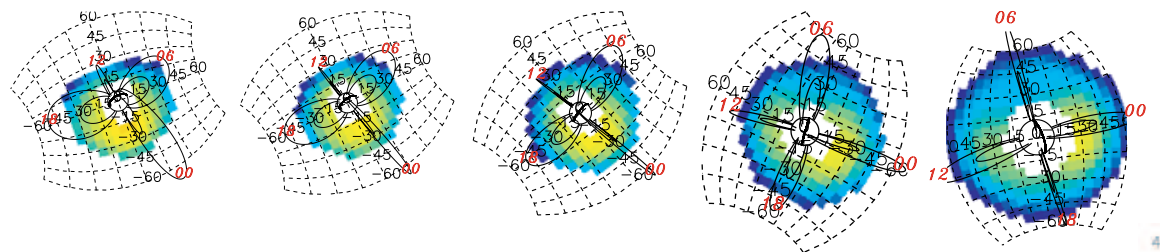

(f) $M=10 @ 16: 00$ (g) $M=10 @ 17: 30$ (h) $M=10 @ 19: 00$ (i) $M=10 @ 20: 30$ (j) $M=10 @ 22: 00$
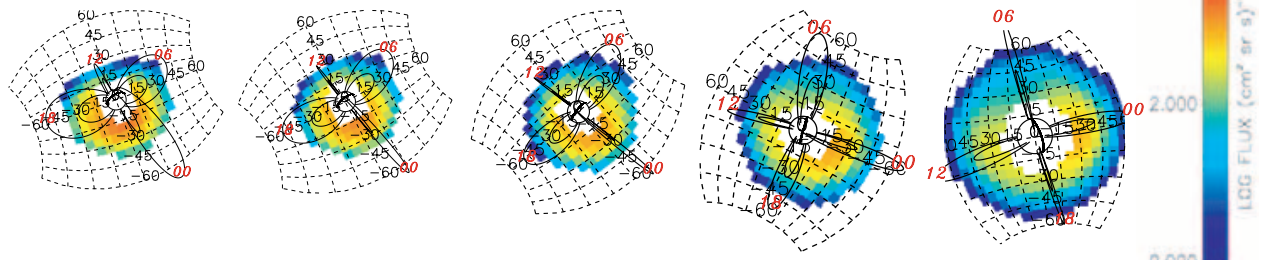

(k) HENA @ 16:00 (l) HENA @ 17:30 (m) HENA @ 19:00 (n) HENA @ 20:30 (o) HENA @ 22:00
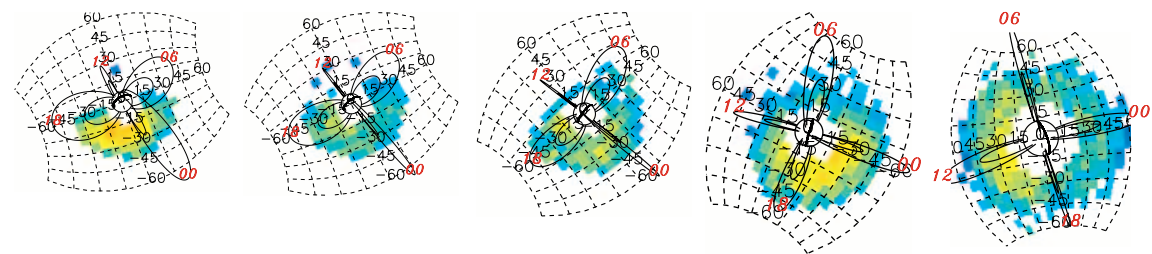

Figure 9. Simulated ENA fluxes for the $M=1$ (top row) and $M=10$ (middle row) simulations at five UTs during the storm (by column: 1600, 1730, 1900, 2030, and 2200). The bottom row shows the corresponding HENA images at the five UTs. The images have been trimmed to show only the spatial region of interest for this study. The format for each plot is the same as in Figures 4d and 4e.

magnitude of the time series (Figure 12a). The plasmapause comparisons are also quite good (Figure 12b), with the same low RMS errors early in the IMAGE orbit followed by an upward trend later on 17 April (Figure 12c). The
ENA flux ratio shows the same decrease halfway through the orbit (Figure 12d), and the ENA flux ratio RMS errors are all very close to each other throughout the storm interval (Figure 12e).

Table 1. Data-Model Comparison Values Averaged Over All UT

\begin{tabular}{|c|c|c|c|c|c|c|c|}
\hline & $D s t *$ Ratio -1 & $D s t * \mathrm{RMS}$ & Ppause Ratio - 1 & Ppause RMS & $\mathrm{F}(39-60)$ Log Ratio & $\mathrm{F}(39-60) \mathrm{RMS}$ & Total Error \\
\hline \multicolumn{8}{|c|}{ FAC-to- $\Sigma$ Relationship } \\
\hline Mult $=1.0$ & -0.25 & 0.28 & 0.22 & 0.41 & -0.10 & 0.52 & 0.33 \\
\hline Mult $=2.5$ & -0.16 & 0.20 & 0.14 & 0.36 & -0.03 & 0.53 & 0.29 \\
\hline Mult $=5.0$ & 0.02 & 0.17 & 0.07 & 0.30 & 0.09 & 0.57 & 0.28 \\
\hline Mult $=7.5$ & 0.08 & 0.20 & 0.04 & 0.31 & 0.13 & 0.60 & 0.29 \\
\hline Mult $=10.0$ & 0.12 & 0.22 & -0.15 & 0.26 & 0.15 & 0.62 & 0.31 \\
\hline \multicolumn{8}{|c|}{ Poleward Oval Shift } \\
\hline Shift $=0.0^{\circ}$ & -0.25 & 0.29 & 0.39 & 0.38 & -0.26 & 0.53 & 0.37 \\
\hline Shift $=2.5$ & -0.07 & 0.17 & 0.14 & 0.33 & 0.01 & 0.54 & 0.27 \\
\hline Shift $=5.0$ & 0.02 & 0.17 & 0.07 & 0.30 & 0.09 & 0.57 & 0.28 \\
\hline Shift $=7.5$ & -0.29 & 0.32 & 0.21 & 0.48 & -0.16 & 0.53 & 0.35 \\
\hline Shift $=10.0$ & -0.39 & 0.42 & 0.28 & 0.43 & -0.28 & 0.54 & 0.40 \\
\hline \multicolumn{8}{|c|}{ Dawnside Equatorward Oval Shift } \\
\hline Tilt $=0.0^{\circ}$ & 0.02 & 0.17 & 0.07 & 0.30 & 0.09 & 0.57 & 0.28 \\
\hline Tilt $=2.5$ & 0.04 & 0.16 & 0.14 & 0.28 & 0.09 & 0.60 & 0.29 \\
\hline Tilt $=5.0$ & -0.04 & 0.15 & -0.16 & 0.30 & 0.03 & 0.59 & 0.29 \\
\hline \multicolumn{8}{|c|}{ High-Latitude Potential Boundary Condition } \\
\hline Weimer-96 & 0.02 & 0.17 & 0.07 & 0.30 & 0.09 & 0.57 & 0.28 \\
\hline V-S Two-cell & -0.08 & 0.18 & 0.14 & 0.31 & 0.01 & 0.54 & 0.27 \\
\hline \multicolumn{8}{|c|}{ Baseline $\Sigma_{P}$} \\
\hline$\Sigma_{\mathrm{P}}=0.1 \mathrm{~S}$ & -0.03 & 0.19 & 0.03 & 0.26 & 0.08 & 0.57 & 0.27 \\
\hline$\Sigma_{\mathrm{P}}=0.5$ & 0.00 & 0.17 & -0.08 & 0.30 & 0.09 & 0.59 & 0.28 \\
\hline$\Sigma_{\mathrm{P}}=1.0$ & 0.02 & 0.17 & 0.07 & 0.30 & 0.09 & 0.57 & 0.28 \\
\hline$\Sigma_{\mathrm{P}}=2.5$ & 0.08 & 0.19 & -0.21 & 0.23 & 0.13 & 0.59 & 0.29 \\
\hline$\Sigma_{\mathrm{P}}=5.0$ & 0.11 & 0.21 & 0.00 & 0.31 & 0.13 & 0.61 & 0.30 \\
\hline
\end{tabular}




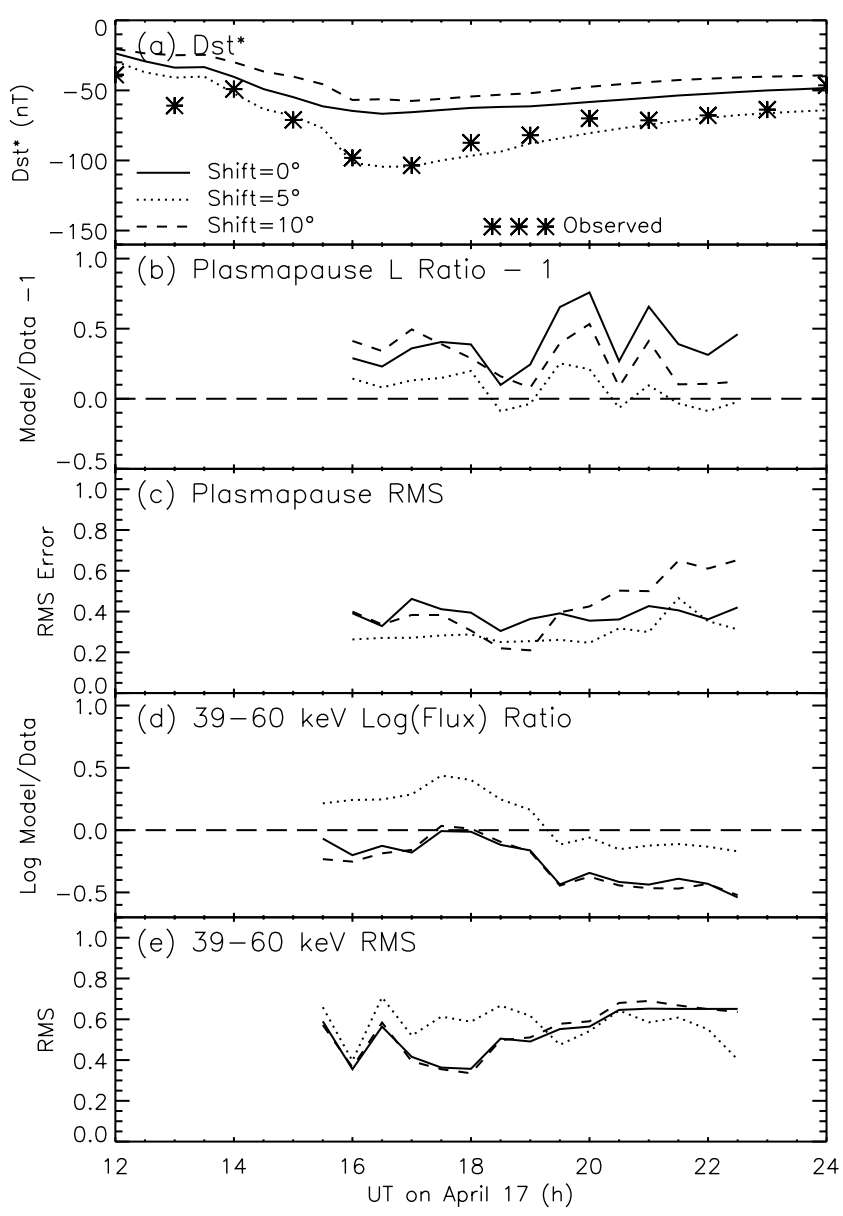

Figure 10. Like Figure 8 except for the simulations with different poleward shifts in the auroral oval conductances relative to the RAM-generated FAC peak. The three lines in each plot show the results for a shift of $0^{\circ}$ (solid line), $5^{\circ}$ (dotted line), and $10^{\circ}$ (dashed line).

[52] Table 1 further demonstrates the similarity between these three dawnside oval tilt settings. The largest difference is seen in the plasmapause $L$ value ratios, where the $T=0^{\circ}$ value is positive and other two values are negative. This can be explained by looking at Figures $5 \mathrm{k}-$ $5 \mathrm{~m}$. The indentation of the predawn plasmapause is smaller when the dawnside location of the oval is tilted equatorward. The higher conductance reduces the electric fields in this region, thus reducing the plasmapause distortion. Figures $7 \mathrm{k}-7 \mathrm{~m}$ show that the nightside potential well is significantly smaller when the oval is tilted equatorward on the dawnside.

[53] Another result seen in Table 1 is that the three simulations have nearly identical total error values, and they are all relatively low (compared to all 16 simulations). In addition, the $D s t^{*} \mathrm{RMS}$ error for the $T=5^{\circ}$ simulation is the lowest of all simulations.

\subsection{High-Latitude Boundary Specification}

[54] Figure 13 shows the data-model comparisons for the simulations with different high-latitude boundary conditions on the electric potential solution. The solid line shows the results when the boundary is specified by the
Weimer-96 empirical model while the dotted line shows the results when a sine-wave (with respect to MLT) is applied, enforcing a Volland-Stern style two-cell convection pattern. Note that the two simulations have the same cross polar cap potential difference; it is just that the potential is arranged differently in local time between the two simulations.

[55] Like Figure 12, the line plots in Figure 13 show that these simulations produce quite comparable results. The lines are quite close together in all of the plots in Figure 13, except perhaps Figure 13c, where the V-S boundary condition produces smaller plasmapause RMS errors early and then larger ones later on. This can be explained by examining Figures $5 \mathrm{n}$ and 50 . The predawn indentation is nonexistent in the V-S boundary condition results (Figure 5o), making the nightside plasmapause locations closer to those extracted from the EUV images. Comparing Figures $7 \mathrm{n}$ and $7 \mathrm{o}$ confirms that it is the absence of the nightside potential well that is responsible for this difference between the simulations. Also absent, however, is the strong electric field region around dusk (the so-called subauroral polarization stream, or SAPS). This results in a miscalculation of the plasmaspheric plume location. When EUV finally measures the dayside plasmasphere later in the orbit, this error manifests itself as an increasing RMS error in Figure 13c.

[56] Figure 14 presents additional inner magnetospheric potential distributions for these two simulations. The nightside potential well associated with the storm-time partial ring current is more pronounced in the Weimer- 96 boundary condition results (top row) than the Volland-Stern two-cell boundary condition results (bottom row), for times throughout the storm sequence (both main phase and recovery phase). Because both simulations include self-consistent electric field calculations, they both produced small-scale potential structures, particularly in the predawn sector. In general, though, there is more small-scale structure in the Weimer-96 boundary condition results. The total potential drop within geosynchronous (that is, the plasma outer boundary location) is also larger for the Weimer-96 boundary condition results. Most of the extra potential difference is located on the duskside, resulting in flow channels that inject the hot ions deeper into the inner magnetosphere than with this concentration of electric field [Chen et al., 2003; Khazanov et al., 2004]. This is a direct result of the high-latitude boundary condition imposed on the electric potential solution; the local time asymmetries present in the Weimer-96 values are translated into the inner magnetosphere.

[57] The reason for this is that the sine-wave boundary condition (in the V-S run) always imposes a spatially uniform (but time-varying) sunward convection throughout the inner magnetosphere. This potential pattern continually pushes the hot ions through the simulation domain, sweeping them out the dayside just as fast as they are swept in the nightside. This reduces the magnitude of the storm-time ring current relative to the simulation with a Weimer-96 boundary condition. This reduction is seen as the slightly smaller values of the $\mathrm{V}-\mathrm{S}$ BC line in Figures 13a and 13d. The smaller hot ion flux intensity is also seen when comparing Figure $6 \mathrm{n}$ with Figure 6o. 

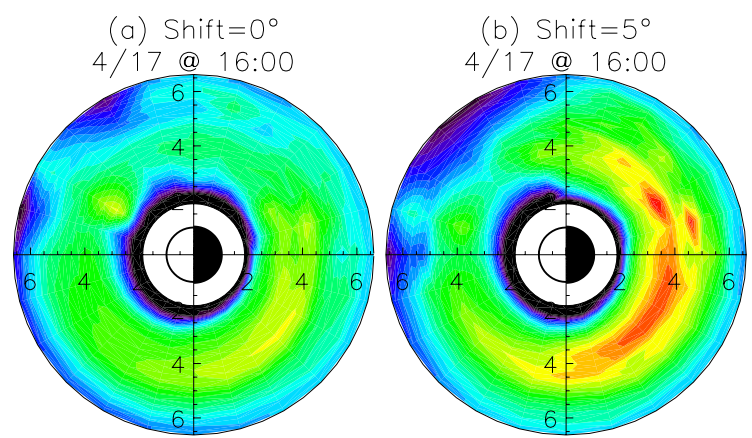

(e) Shift $=5^{\circ}$

(d) Shift $=0^{\circ}$
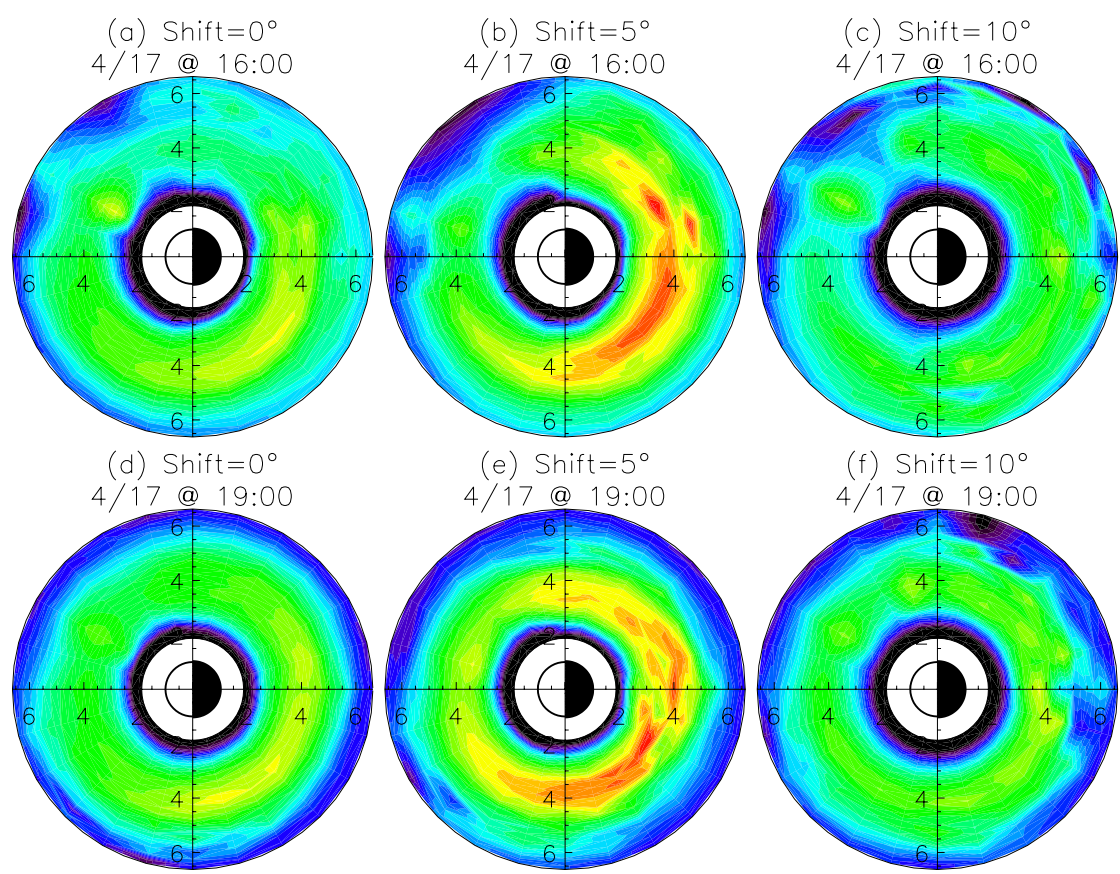

(f) Shift $=10^{\circ}$

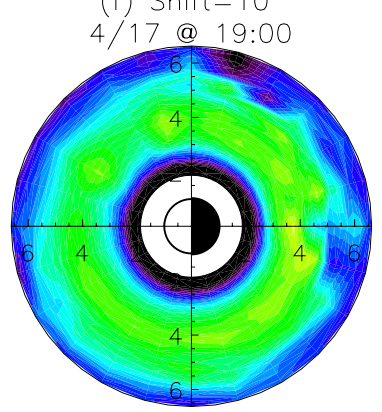

Figure 11. Hot ion pressures $\left(1-400 \mathrm{keV}, \mathrm{H}^{+}\right.$and $\left.\mathrm{O}^{+}\right)$in the inner magnetosphere for $\mathrm{S}=0,5$, and $10^{\circ}$ (the three columns, respectively) at 1600 and 1900 UT (upper and lower rows, respectively). The format is the same as Figure 4c, and the pressures are on a logarithmic color scale.

[58] Table 1 shows the integrated error values for these two simulations, relative to the chosen data sets. It is seen that the Weimer-96 boundary condition run did better at predicting $D s t^{*}$ and the plasmapause location, but the Volland-Stern two-cell boundary condition run was better at predicting the $39-60 \mathrm{keV}$ hot ion fluxes. The total errors of the two simulations are very close to each other, but the V-S result is just slightly better. In fact, it is tied with two other simulations for the best overall error value.

\subsection{Baseline "Starlight $\Sigma$ " Setting}

[59] Figure 15 shows the data-model comparisons for the simulations with different baseline Pedersen conductance settings (the starlight $\Sigma_{\mathrm{P}}$ setting). The lines in each plot show the results for values of $\Sigma_{\mathrm{P}}=0.1 \mathrm{~S}, 1.0 \mathrm{~S}$, and $5.0 \mathrm{~S}$ (solid, dotted, and dashed lines, respectively). Note that the oval is shifted poleward $5^{\circ}$ for all of these simulations.

[60] The three simulations produce very similar datamodel comparisons. The trend is that a higher $\Sigma_{\mathrm{P}}$ setting yields a slightly stronger ring current. Changing the baseline value by a factor of 50 produced a $-15 \mathrm{nT}$ deeper depression in $\Delta B_{\mathrm{DPS}}$ (Figure 15a). The differences in the other four panels of Figure 15 are essentially negligible. The similarity between these simulations is also seen in the plasmapause location comparisons in Figures $5 p-5 t$ and the ENA flux ratio images in Figures $6 \mathrm{p}-6 \mathrm{t}$.

[61] Table 1 also shows the similarity between these simulations. The trends are summarized in the total error column, showing that the $\Sigma_{\mathrm{P}}=0.1 \mathrm{~S}$ setting yielded the best comparison with the data, and the others compared slightly less well in order of increasing $\Sigma_{\mathrm{p}}$. Four numbers in this portion of Table 1 are the best within their column: the Dst* ratio for the $\Sigma_{\mathrm{P}}=0.5 \mathrm{~S}$ setting; the plasmapause ratio for the $\Sigma_{\mathrm{P}}=5.0 \mathrm{~S}$ setting; the plasmapause RMS error for the $\Sigma_{\mathrm{P}}=2.5 \mathrm{~S}$ setting; and the total error value (tied with two others) for the $\Sigma_{\mathrm{P}}=0.1 \mathrm{~S}$ setting.

\section{Discussion}

[62] A consistent trend throughout all of the results presented above is that there is an optimal conductance intensity for maximum ring current generation. If the nightside subauroral conductance is too low, then the injection of any plasma into the inner magnetosphere creates large potential structures (and therefore strong electric fields) that act to not only break up the injected pressure peak but also inhibit additional injections from the plasma sheet. As the conductance is increased, these electric fields are reduced, allowing for a larger storm-time ring current. If the conductance gets too large, however, then the inner magnetospheric electric fields become small. That is, the potential drop must occur elsewhere, i.e., outside of geosynchronous orbit. This has two detrimental effects on the storm-time ring current intensity: injections from the plasma sheet do not penetrate as deeply, reducing the adiabatic energization of the hot ions; and the shielding field is reduced, so the large-scale (and sunward) magnetospheric electric field extends throughout the region and the near-Earth hot ions are efficiently swept out toward the dayside magnetopause. This is most evident in the results presented in section 4.2 (poleward oval shift variations), but the other parameters also exhibit one extreme or the other of this trend.

[63] Another consistent feature of the data-model comparisons is that the observed and modeled ENA flux distributions are different at the very end of the IMAGE orbit (see the last two columns of Figure 9). One explanation for this is that the particles drifted from the nightside to 


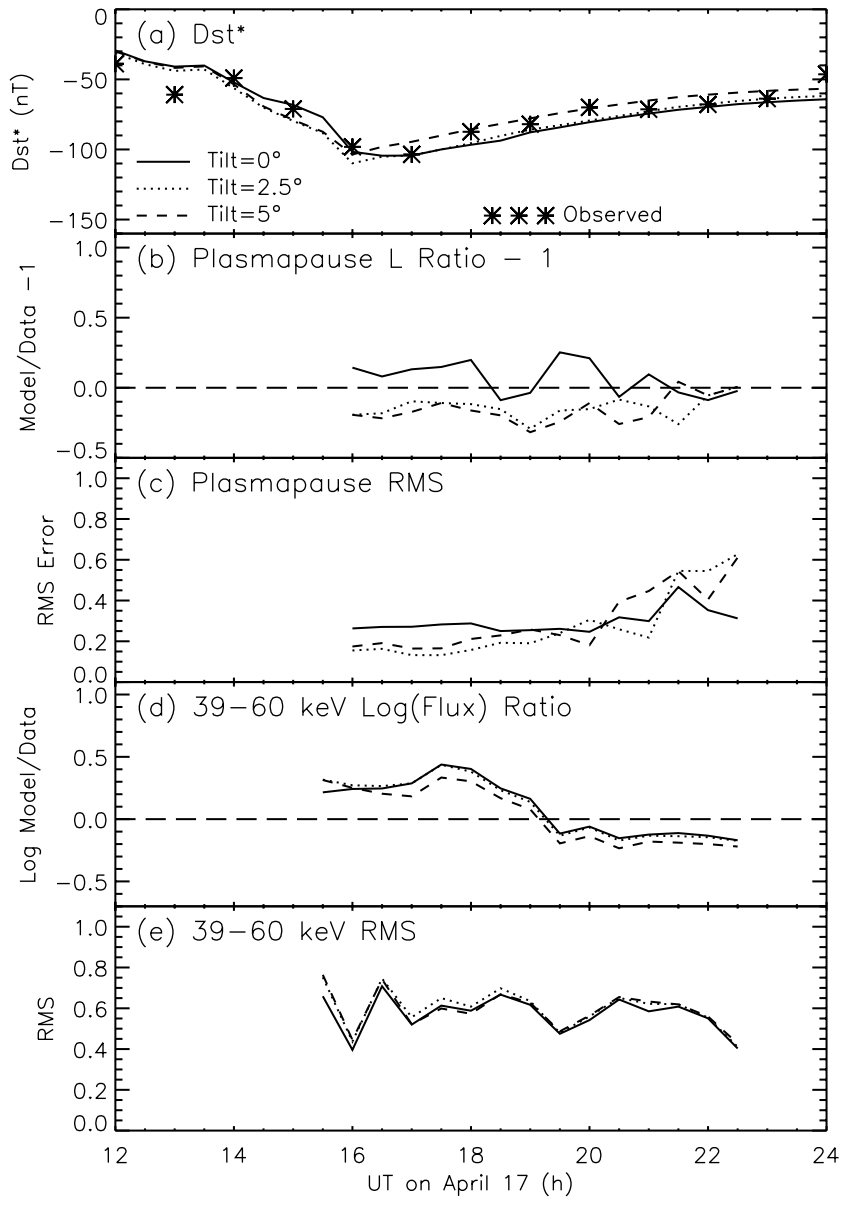

Figure 12. Like Figure 8 except for the simulations with different dawnside-only equatorward shifts in the auroral oval conductances relative to the duskside latitude. The three lines in each plot show the results for a tilt of $0^{\circ}$ (solid line), $2.5^{\circ}$ (dotted line), and $5^{\circ}$ (dashed line).

the dayside during the interval from 2030 to 2200 and that this shift in the peak location was not predicted by any of the RAM simulations. Assuming that the ion peak is at $L=$ 4 (see Figures 4 and 11), a rotation of 10 hours in MLT over 1.5 hours of UT yields a drift speed of $12.4 \mathrm{~km} / \mathrm{s}$. The magnetic gradient-curvature drift of a $50 \mathrm{keV}$ ion at $L=4$ in a dipole field is $12.2 \mathrm{~km} / \mathrm{s}$. It appears that magnetic drift alone can explain the westward shift in the plasma pressure peak.

[64] The reason that this shift is not seen in the RAMgenerated ENA flux images is because fresh plasma sheet ions are being injected throughout the recovery phase of the storm. Consider the potential patterns shown in Figure 14. Westward electric fields (which produce radially inward plasma drift) are present across the nightside, in to $L=4$, even at times as late as 2200 UT on 17 April. These electric fields continue to supply the inner magnetosphere with plasma sheet ions, thus refreshing the nightside pressure peak throughout the recovery phase of the storm. The ENA flux discrepancy implies that this ongoing injection of plasma into the inner magnetosphere occurs until 2030 but then shuts off at this time. A possible reason for such a convection ramp-down is discussed below.
[65] Another possible reason for the ENA flux discrepancy is that the observed pitch angle, which changes as IMAGE progresses along its orbit track, has different flux values relative to the PA-averaged value used in these plots. The observed pitch angle at each spatial location in the inner magnetosphere is highly dependent on the viewing geometry, and the satellite is moving rapidly over the pole and down toward the planet during this interval. The forward modeling software used for this analysis, however, assumes pitch angle isotropy for the hot ions, and so pitch angle averaged flux values from RAM were used to generate the simulated ENA images. The general trend is that as the satellite moves from equator to over the pole, the observed pitch angle changes from large values to small ones. Therefore it could be that the continual injection predicted by RAM is still occurring but that the particles injected after 2030 are highly anisotropic.

[66] The plausibility of these two explanations can be assessed by examining the available in situ data for this interval. There are two LANL geosynchronous satellites on the nightside during the final hours of 17 April 2002, located premidnight and postmidnight (LANL-01a and

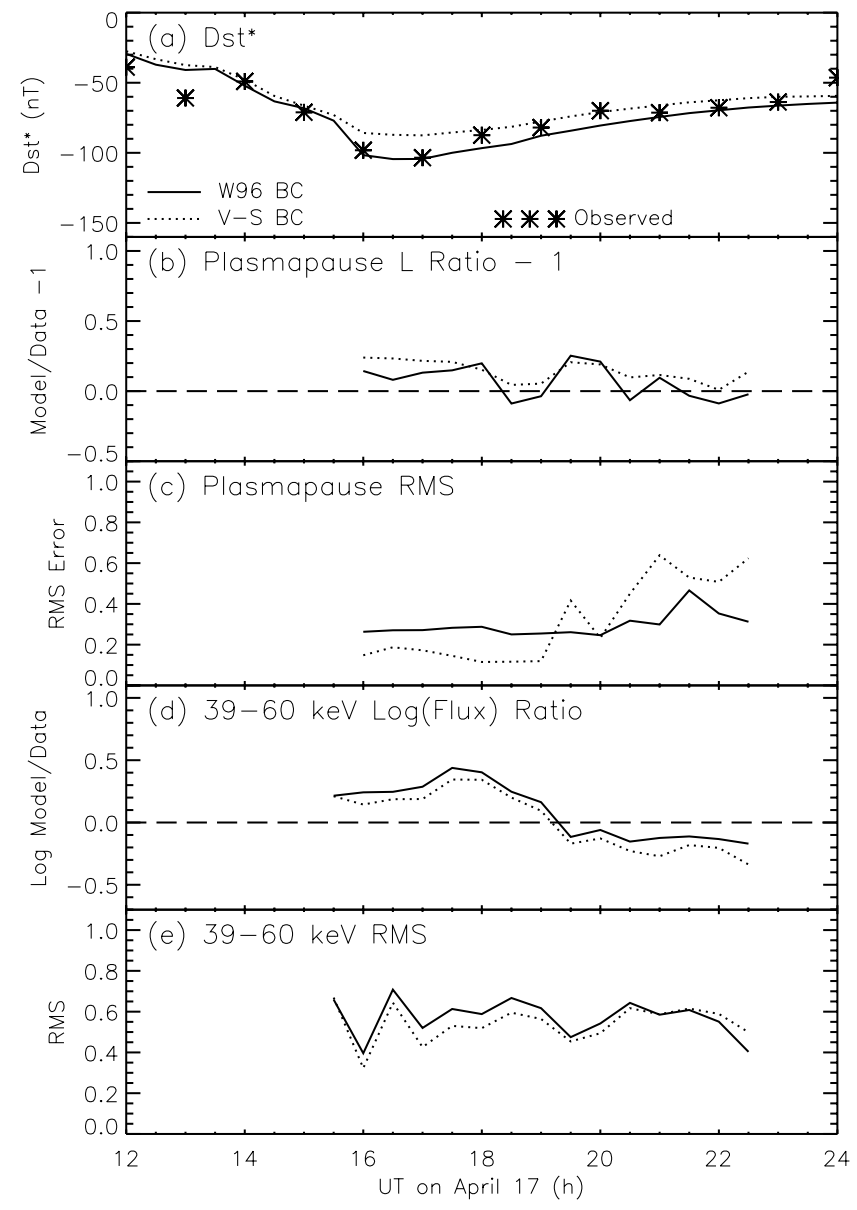

Figure 13. Like Figure 8 except for the simulations with different high latitude boundary condition specifications for the potential calculation. The two lines in each plot show the results for a Weimer-96 boundary condition (solid line) and for a Volland-Stern 2-cell pattern boundary condition (dotted line). 
(a) $\mathrm{W}-96 \mathrm{BC}$

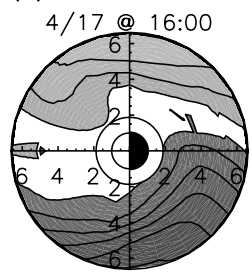

(f) $\mathrm{V}-\mathrm{S} B \mathrm{BC}$

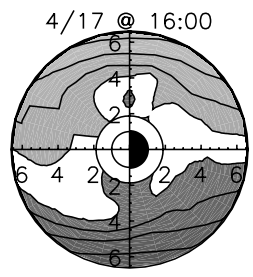

(b) $\mathrm{W}-96 \mathrm{BC}$

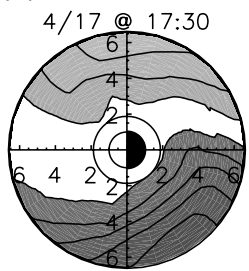

(g) $\mathrm{V}-\mathrm{S} B \mathrm{BC}$

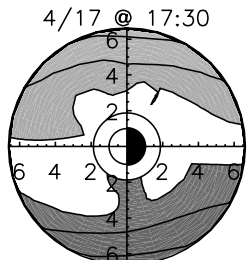

(c) $\mathrm{W}-96 \mathrm{BC}$

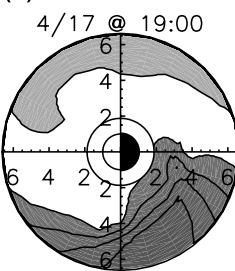

(h) $\mathrm{V}-\mathrm{S} B \mathrm{BC}$

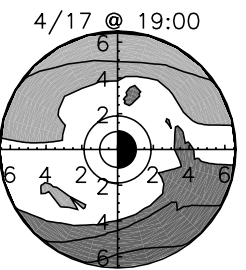

Well (d) $\mathrm{W}-96 \mathrm{BC}$

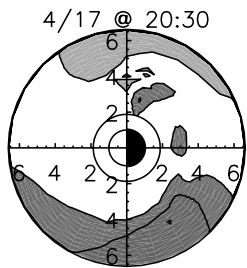

(i) $\mathrm{V}-\mathrm{S} \mathrm{BC}$

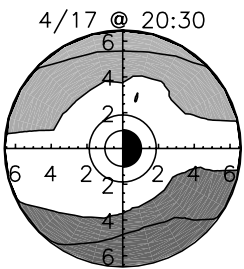

Peak (e) $\mathrm{W}-96 \mathrm{BC}$

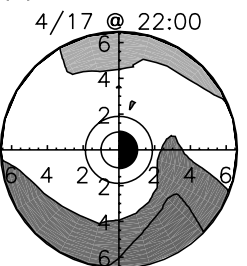

(j) $V-S B C$

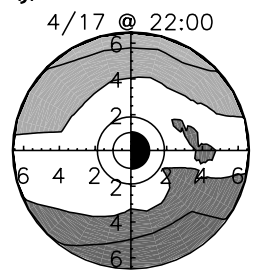

Figure 14. Electric potential contours for the Weimer-96 and Volland-Stern high-latitude boundary condition simulations (upper and lower rows, respectively) at five UTs during the storm (by column: 1600, 1730, 1900, 2030, and 2200). The format is the same as in Figure 7.

LANL-02a are at local midnight at 2333 and 1921, respectively). Both measure isotropic hot ion temperature distributions, which is strong evidence against this second interpretation. However, the hot ion densities are relatively constant from 1830 to the end of the day (between 1 and $2 \mathrm{~cm}^{-3}$ for all but a few minutes, as seen in Figure 1d). In addition, there was a relatively steady cross polar cap potential from 1900 to 2200 (varies between 80 and $120 \mathrm{kV}$, as seen in Figure 1e). Because these two parameters are the most critical factors in determining the ring current intensity [e.g., Kozyra and Liemohn, 2003], their steadiness and similarity before and after 2030 is evidence against the first explanation.

[67] A possible resolution is that the high-latitude boundary condition on the potential, whether from Weimer-96 or a sine-wave function, does not reflect the real potential distribution during this interval. The large-scale magnetospheric convection field might have greatly weakened around 2030 (for some reason), effectively shutting off the inflow of plasma sheet particles after this time. There is, however, no strong evidence for a change in the convection pattern at this time, either in the solar wind data (see Figures $1 \mathrm{~b}$ and $1 \mathrm{c}$ ) or in the IMAGE FUV images (not shown). Therefore it is unclear why the HENA image sequence disagrees with the RAM-generated simulated ENA images late on 17 April.

[68] Note that there was a substorm onset (seen in the FUV/WIC images) at 1904 UT. Goldstein et al. [2005] and Brandt et al. [2005] describe this substorm in detail, concluding that an electric field pulse surged through the inner magnetosphere during the expansion phase. It could be that the large-scale electric field did not resume after the pulse propagated through the magnetosphere. The largescale convection ramp-down just after the substorm could be coincidental or it could be that the substorm-induced pulse altered the region 2 currents that maintain the inner

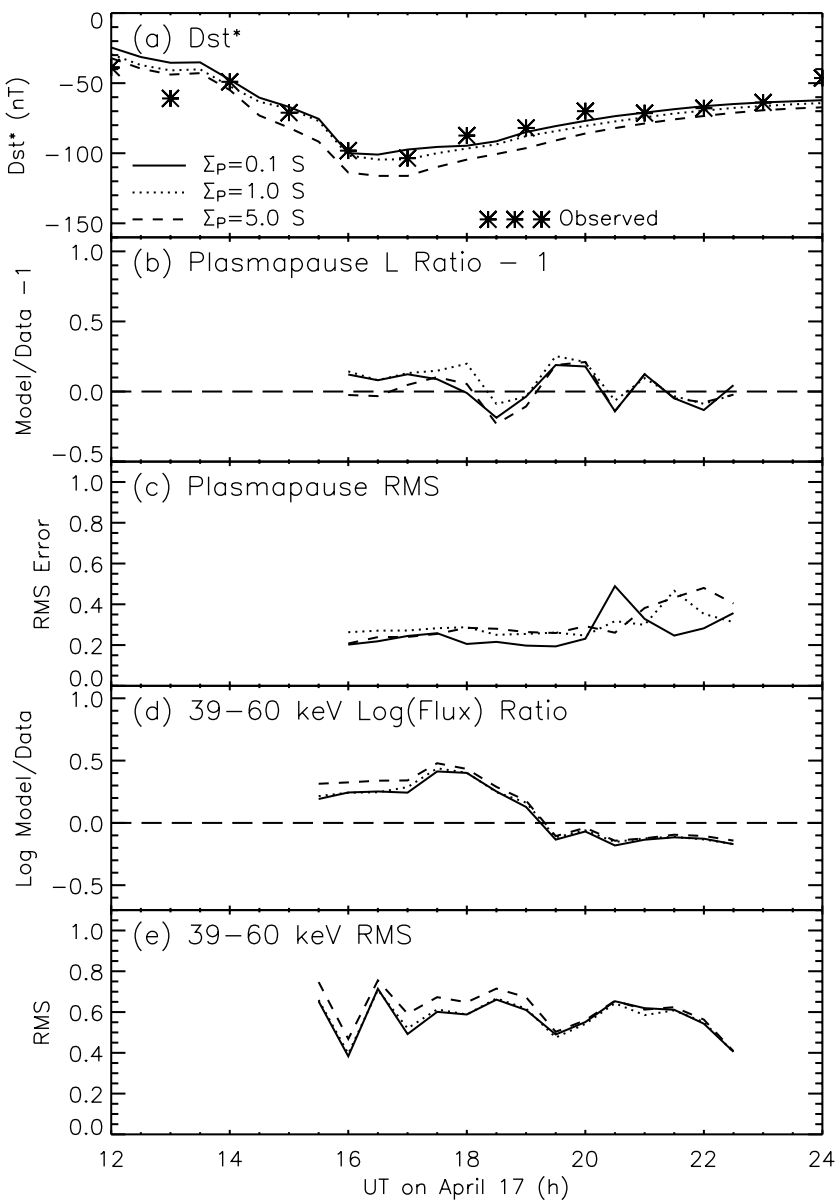

Figure 15. Like Figure 8 except for the simulations with different baseline Pedersen conductance values. The three lines in each plot show the results for a baseline value of 0.1 S (solid line), 1.0 S (dotted line), and 5.0 S (dashed line). 
magnetospheric electric field morphology (in particular the strong subauroral westward flow in the evening and dusk sectors). A detailed examination of any relationship between the substorm and the convection ramp-down is left for a future study.

\section{Conclusions}

[69] Numerous simulations were conducted in order to quantify the influence of nightside conductance morphology and intensity on the dynamics of the inner magnetosphere. The RAM ring current simulation code was used, along with the DGCPM plasmasphere code and an ionospheric potential solver. The study focused on the moderate magnetic storm of 17 April 2002. The simulation results were compared against measurements of the ring current and plasmasphere in order to assess the accuracy as a function of conductance parameter setting. In particular, three data sets were used: $D s t^{*}$, plasmapause location as extracted from IMAGE EUV snapshots, and IMAGE HENA flux observations for the $39-60 \mathrm{keV}$ energy range.

[70] No single simulation conducted for this study proved itself to be the best overall match to the selected data sets. Many things were learned from the simulations, however. The major findings of this study are as follows:

[71] 1. There is an optimal conductance level for maximal ring current intensity. Too little conductance leads to large shielding potentials that effectively inhibit ring current growth, while too much conductance leads to continual flow-through of the hot ions with little hot ion accumulation in the inner magnetosphere.

[72] 2. The selection of an optimal conductance was most dramatically seen in the simulations varying the poleward shift of the auroral conductance oval relative to the RAMgenerated FACs. A poleward offset of $2.5^{\circ}$ or $5^{\circ}$ was the best at reproducing the data.

[73] 3. Increasing the intensity of the auroral zone conductance (from a peak value of $\sim 5 \mathrm{~S}$ up to a value of $\sim 50 \mathrm{~S}$ ) increased the ring current intensity. A peak value of $\sim 25 \mathrm{~S}$ ( $M=5$ setting) was the best at reproducing the data.

[74] 4. A dawnside equatorward tilt of the auroral zone (relative to its duskside latitude) was found to have little effect. The only discernible trend was that more tilt slightly reduced the size of the nightside potential well and therefore the eastward MLT shift of the hot ion pressure peak.

[75] 5. The choice of high-latitude boundary condition on the potential solution had little effect on the data-model comparisons. The biggest difference was that a sine-wave boundary condition resulted in a weaker nightside potential well. That is, specific features of the inner magnetospheric electric field are dependent on the local time distribution of the high-latitude potential, but the overall data-model error was very similar between these simulations.

[76] 6. Increasing the uniformly-applied baseline conductance (from $0.1 \mathrm{~S}$ to $5 \mathrm{~S}$ ) had little influence on the datamodel comparisons. The trend was that a higher baseline resulted in a slightly more intense ring current.

[77] 7. The westward drift of the ENA flux peak from 2030 UT to 2200 UT cannot be definitively explained, although several candidate reasons are listed in the section above.
[78] The results of this study are complementary to several other recent studies examining ionospheric conductance effects on the ring current. Most notably, Ebihara et al. [2004] conducted an assessment very similar to this one, but for the intense storm of 12 August 2000 (Dst minimum was $-235 \mathrm{nT}$, compared to $-98 \mathrm{nT}$ for the 17 April 2002 storm examined in the present study). They concentrated on dayside conductance influences (varying $F_{10.7}$ and day of year), with auroral zone conductance influences limited to a comparison of two descriptions (the Hardy et al. [1987] empirical model and conductance extractions from IMAGE FUV observations). They concluded that brief auroral brightenings (that is, transient increases in auroral zone conductance) do not significantly affect the ring current intensity. The results of the present study extend that finding: long-duration auroral conductance increases do influence ring current intensity (see Figures $6 a-6 e$ and Figure 9).

[79] Another relevant study is that of Ebihara and Fok [2004], who performed numerous simulations and compared the results against IMAGE HENA images for the 39-50 keV range (for several magnetic storms). Their primary concern was in describing the physical mechanisms that control the MLT location of the flux peak. The results presented here agree with the Ebihara and Fok [2004] finding that the poleward shift of the auroral oval relative to the region-2 FACs does not change the MLT location of the flux peak (see Figures $6 \mathrm{f}-6 \mathrm{j}$ and Figure 11). The peak intensity of the ring current, however, is highly dependent on the poleward shift of the oval. In fact, this was the parameter with the biggest influence on the data-model comparisons.

[80] Acknowledgments. The authors would like to thank the sources of funding for this study: NASA grants NAG5-10297, NAG-10850, and NAG-12772 and NSF grants ATM-0090165 and ATM-0302529. The authors would also like to thank all of their data providers who made the ring current simulations possible, especially M. F. Thomsen and G. D. Reeves at the Los Alamos National Laboratory, the Kyoto World Data Center for the $K p$ and Dst index, and CDAWeb for allowing access to the level-2 plasma and magnetic field data of the ACE spacecraft (and D. J. McComas and N. Ness for providing their data to CDAWeb).

[81] Arthur Richmond thanks Yusuke Ebihara and another reviewer for their assistance in evaluating this paper.

\section{References}

Bame, S. J., et al. (1993), Magnetospheric plasma analyzer for spacecraft with constrained resources, Rev. Sci. Instrum., 64, 1026.

Belian, R. D., G. R. Gisler, T. Cayton, and R. Christensen (1992), High-Z energetic particles at geosynchronous orbit during the great solar proton event series of October 1989, J. Geophys. Res., 97, 16,897.

Brandt, P. C., S. Ohtani, D. G. Mitchell, R. Demajistre, and E. C. Roelof (2002a), ENA observations of a global substorm growth phase dropout in the nightside magnetosphere, Geophys. Res. Lett., 29(20), 1962, doi:10.1029/2002GL015057.

Brandt, P. C., R. Demajistre, E. C. Roelof, S. Ohtani, D. G. Mitchell, and S. Mende (2002b), IMAGE/high-energy energetic neutral atom: Global energetic neutral atom imaging of the plasma sheet and ring current during substorms, J. Geophys. Res., 107(A12), 1454, doi:10.1029/ 2002JA009307.

Brandt, P. C., S. Ohtani, D. G. Mitchell, M.-C. Fok, E. C. Roelof, and R. Demajistre (2002c), Global ENA observations of the storm mainphase ring current: Implications for skewed electric fields in the inner magnetosphere, Geophys. Res. Lett., 29(20), 1954, doi:10.1029/ 2002GL015160.

Brandt, P. C., D. G. Mitchell, Y. Ebihara, B. R. Sandel, E. C. Roelof, J. L. Burch, and R. Demajistre (2002d), Global IMAGE/HENA observations of the ring current: Examples of rapid response to IMF and ring current-plasmasphere interaction, J. Geophys. Res., 107(A11), 1359, doi:10.1029/2001JA000084 
Brandt, P. C., J. Goldstein, B. J. Anderson, H. Korth, T. J. Immel, E. C. Roelof, R. DeMajistre, D. G. Mitchell, and B. R. Sandel (2005), On the relation between electric fields in the inner magnetosphere, ring current, auroral conductance, and plasmapause motion, in Inner Magnetosphere Interactions: New Perspectives From Imaging, Geophys. Monogr. Ser, vol. 159, edited by J. L. Burch, M. Schulz, and H. Spence, p. 153, AGU, Washington, D. C.

Burch, J. L. (2000), IMAGE mission overview, Space Sci. Rev., 91, 1.

Burch, J. L. (2003), The first two years of IMAGE, Space Sci. Rev., 109, 1.

Carovillano, R. L., and G. L. Siscoe (1973), Energy and momentum theorems in magnetospheric processes, Rev. Geophys., 11, 289.

Carpenter, D. L. (1963), Whistler evidence of a "knee" in the magnetospheric ionization density profile, J. Geophys. Res., 68, 1675.

Carpenter, D. L., and R. R. Anderson (1992), An ISEE/whistler model of equatorial electron density in the magnetosphere, J. Geophys. Res., 97, 1097.

Chen, M. W., M. Schulz, G. Lu, and L. R. Lyons (2003), Quasi-steady drift paths in a model magnetosphere with AMIE electric field: Implications for ring current formation, J. Geophys. Res., 108(A5), 1180, doi:10.1029/ 2002JA009584

Daglis, I. A., R. M. Thorne, W. Baumjohann, and S. Orsini (1999), The terrestrial ring current: Origin, formation and decay, Rev. Geophys., 37, 407

DeMajistre, R. E., C. Roelof, P. C. Brandt, and D. G. Mitchell (2004), Retrieval of global magnetospheric ion distributions from high-energy neutral atom measurements made by the IMAGE/HENA instrument, J. Geophys. Res., 109, A04214, doi:10.1029/2003JA010322.

Dessler, A. J., and E. N. Parker (1959), Hydromagnetic theory of geomagnetic storms, J. Geophys. Res., 64, 2239.

Ebihara, Y., and M. Ejiri (2002), Numerical simulation of the ring current: A review, Space Sci. Rev., 105, 377.

Ebihara, Y., and M.-C. Fok (2004), Postmidnight storm-time enhancement of tens-of-keV proton flux, J. Geophys. Res., 109, A12209, doi:10.1029/ 2004JA010523.

Ebihara, Y., M.-C. Fok, R. A. Wolf, T. J. Immel, and T. E. Moore (2004), Influence of ionospheric conductivity on the ring current, J. Geophys. Res., 109, A08205, doi:10.1029/2003JA010351.

Ebihara, Y., M.-C. Fok, R. A. Wolf, M. F. Thomsen, and T. E. Moore (2005), Nonlinear impact of plasma sheet density on the storm-time ring current, J. Geophys. Res., 110, A02208, doi:10.1029/2004JA010435.

Fok, M.-C., J. U. Kozyra, A. F. Nagy, C. E. Rasmussen, and G. V. Khazanov (1993), A decay model of equatorial ring current and the associated aeronomical consequences, J. Geophys. Res., 98, 19,381.

Fok, M.-C., R. A. Wolf, R. W. Spiro, and T. E. Moore (2001), Comprehensive computational model of the Earth's ring current, J. Geophys. Res., 106, 8417

Fok, M.-C., et al. (2003), Global ENA image simulations, Space Sci. Rev., 109, 77

Frank, L. A. (1970), Direct detection of asymmetric increases of extraterrestrial "ring current" proton intensities in the outer radiation zone J. Geophys. Res., 75, 1263.

Galand, M., and A. D. Richmond (2001), Ionospheric electrical conductances produced by auroral proton precipitation, J. Geophys. Res., 106 117

Garner, T. W. (2003), Numerical experiments on the inner magnetospheric electric field, J. Geophys. Res., 108(A10), 1373, doi:10.1029 2003JA010039.

Goldstein, J., R. W. Spiro, P. H. Reiff, R. A. Wolf, B. R. Sandel, J. W. Freeman, and R. L. Lambour (2002), IMF-driven overshielding electric field and the origin of the plasmaspheric shoulder on May 24, 2000, Geophys. Res. Lett., 29(16), 1819, doi:10.1029/2001GL014534.

Goldstein, J., B. R. Sandel, M. R. Hairston, and P. H. Reiff (2003), Control of plasmaspheric dynamics by both convection and sub-auroral polarization stream, Geophys. Res. Lett., 30(24), 2243, doi:10.1029/ 2003GL018390.

Goldstein, J., R. A. Wolf, B. R. Sandel, and P. H. Reiff (2004), Electric fields deduced from plasmapause motion in IMAGE EUV images, Geophys. Res. Lett., 31, L01801, doi:10.1029/2003GL018797.

Goldstein, J., J. L. Burch, B. R. Sandel, S. B. Mende, P. C. Brandt, and M. R. Hairston (2005), Coupled response of the inner magnetosphere and ionosphere on 17 April 2002, J. Geophys. Res., 110, A03205, doi:10.1029/2004JA010712.

Grafe, A. (1999), Are our ideas about Dst correct?, Ann. Geophys., 17, 1.

Greenspan, M. E., and D. C. Hamilton (2000), A test of the DesslerParker-Sckopke relation during magnetic storms, J. Geophys. Res., 105, 5419

Hardy, D. A., M. S. Gussenhoven, R. Raistrick, and W. J. McNeil (1987), Statistical and functional representations of the pattern of auroral energy flux, number flux, and conductivity, J. Geophys. Res., $92,12,275$.
Iijima, T., and T. A. Potemra (1976), The amplitude distribution of fieldaligned currents at northern latitudes observed by TRIAD, J. Geophys. Res., 81, 2165.

Iyemori, T. (1990), Storm-time magnetospheric currents inferred from midlatitude geomagnetic field variations, J. Geomagn. Geoelectr., 42, 1249.

Jaggi, R. K., and R. A. Wolf (1973), Self-consistent calculation of the motion of a sheet of ions in the magnetosphere, J. Geophys. Res., 78, 2842 .

Jordanova, V. K., L. M. Kistler, J. U. Kozyra, G. V. Khazanov, and A. F. Nagy (1996), Collisional losses of ring current ions, J. Geophys. Res., $101,111$.

Jordanova, V. K., A. Boonsiriseth, R. M. Thorne, and Y. Dotan (2003), Ring current asymmetry from global simulations using a high-resolution electric field model, J. Geophys. Res., 108(A12), 1443, doi:10.1029/ 2003JA009993.

Jorgensen, A. M., H. E. Spence, W. J. Hughes, and H. J. Singer (2004), A statistical study of the global structure of the ring current, J. Geophys. Res., 109, A12204, doi:10.1029/2003JA010090.

Khazanov, G. V., M. W. Liemohn, T. S. Newman, M.-C. Fok, and R. W. Spiro (2003), Self-consistent magnetosphere-ionosphere coupling: Theoretical studies, J. Geophys. Res., 108(A3), 1122, doi:10.1029/ 2002JA009624.

Khazanov, G. V., M. W. Liemohn, T. S. Newman, M.-C. Fok, and A. J. Ridley (2004), Magnetospheric convection electric field dynamics and stormtime particle energization: Case study of the magnetic storm of 4 May 1998, Ann. Geophys., 22, 497.

Kozyra, J. U., and M. W. Liemohn (2003), Ring current energy input and decay, Space Sci. Rev., 109, 105.

Kozyra, J. U., M. W. Liemohn, C. R. Clauer, A. J. Ridley, M. F. Thomsen, J. E. Borovsky, J. L. Roeder, and V. K. Jordanova (2002), Two-step Dst development and ring current composition changes during the 4-6 June 1991 magnetic storm, J. Geophys. Res., 107(A8), 1224, doi:10.1029/ 2001JA000023.

Lemaire, J. F., and K. I. Gringauz (1998), The Earth's Plasmasphere, Cambridge Univ. Press, New York.

Liemohn, M. W. (2003), Yet another caveat to the Dessler-Parker-Sckopke relation, J. Geophys. Res., 108(A6), 1251, doi:10.1029/2003JA009839.

Liemohn, M. W., and P. C. Brandt (2005), Small-scale structure in the stormtime ring current, in Inner Magnetosphere Interactions: New Perspectives From Imaging, Geophys. Monogr. Ser., vol. 159, edited by J. L. Burch, M. Schulz, and H. Spence, p. 167, AGU, Washington, D. C.

Liemohn, M. W., and J. U. Kozyra (2003), Lognormal form of the ring current energy content, J. Atmos. Sol. Terr. Phys., 65, 871.

Liemohn, M. W., J. U. Kozyra, M. F. Thomsen, J. L. Roeder, G. Lu, J. E. Borovsky, and T. E. Cayton (1999a), Dominant role of the asymmetric ring current in producing the stormtime Dst*, J. Geophys. Res., 106, 10,883 .

Liemohn, M. W., J. U. Kozyra, V. K. Jordanova, G. V. Khazanov, M. F. Thomsen, and T. E. Cayton (1999b), Analysis of early phase ring current recovery mechanisms during geomagnetic storms, Geophys. Res. Lett., $25,2845$.

Liemohn, M. W., J. U. Kozyra, C. R. Clauer, and A. J. Ridley (2001), Computational analysis of the near-Earth magnetospheric current system, J. Geophys. Res., 106, 29,531.

Liemohn, M. W., A. J. Ridley, D. L. Gallagher, D. M. Ober, and J. U. Kozyra (2004), Dependence of plasmaspheric morphology on the electric field description during the recovery phase of the April 17, 2002 magnetic storm, J. Geophys. Res., 109, A03209, doi:10.1029/2003JA010304. McComas, D. J., S. J. Bame, P. Barker, W. C. Feldman, J. L. Phillips, P. Riley, and J. W. Griffee (1998), Solar wind electron proton alpha monitor (SWEPAM) for the Advanced Composition Explorer, Space Sci. Rev., 86, 563

Mitchell, D. G., et al. (2000), High energy neutral atom (HENA) imager for the IMAGE mission, Space Sci. Rev., 91, 67.

Mitchell, D. G., K. C. Hsieh, C. C. Curtis, D. C. Hamilton, H. D. Voss, E. C. Roelof, and P. Brandt (2001), Imaging two geomagnetic storms in energetic neutral atoms, Geophys. Res. Lett., 28, 1151.

Mitchell, D. G., P. C. Brandt, E. C. Roelof, D. C. Hamilton, K. C. Retterer, and S. Mende (2003), Global imaging of $\mathrm{O}^{+}$from IMAGE/HENA, Space Sci. Rev., 109, 63

Ober, D. M., J. L. Horwitz, and D. L. Gallagher (1997), Formation of density troughs embedded in the outer plasmasphere by subauroral ion drift events, J. Geophys. Res., 102, 14,595.

Parker, E. N. (1957), Newtonian development of the dynamical properties of ionized gases of low density, Phys. Rev., 107, 924.

Rairden, R. L., L. A. Frank, and J. D. Craven (1986), Geocoronal imaging with Dynamics Explorer, J. Geophys. Res., 91, 13,613.

Ridley, A. J., and M. W. Liemohn (2002), A model-derived description of the penetration electric field, J. Geophys. Res., 107(A8), 1151, doi:10.1029/2001JA000051. 
Ridley, A. J., D. L. De Zeeuw, T. I. Gombosi, and K. G. Powell (2001), Using steady-state MHD results to predict the global state of the magnetosphere-ionosphere system, J. Geophys. Res., 106, 30,067.

Ridley, A. J., T. I. Gombosi, and D. L. De Zeeuw (2004), Ionospheric control of the magnetosphere: Conductance, Ann. Geophys., 22, 567.

Sandel, B. R., et al. (2000), The extreme ultraviolet imager investigation for the IMAGE mission, Space Sci. Rev, 91, 197.

Sandel, B. R., J. Goldstein, D. L. Gallagher, and M. Spasojevic (2003), Extreme ultraviolet imager observations of the structure and dynamics of the plasmasphere, Space Sci. Rev., 109, 25.

Sazykin, S., R. A. Wolf, R. W. Spiro, T. I. Gombosi, D. L. De Zeeuw, and M. F. Thomsen (2002), Interchange instability in the inner magnetosphere associated with geosynchronous particle flux decreases, Geophys. Res. Lett., 29(10), 1448, doi:10.1029/2001GL014416.

Sckopke, N. (1966), A general relation between the energy of trapped particles and the disturbance field near the Earth, J. Geophys. Res., 71, 3125

Singer, S. F. (1957), A new model of magnetic storms and aurorae, Eos Trans. $A G U, 38,175$

Smith, C. W., M. H. Acuña, L. F. Burlaga, J. L'Heureux, N. F. Ness, and J. Scheifele (1998), The ACE magnetic fields experiment, Space Sci Rev., 86, 613.

Southwood, D. J., and R. A. Wolf (1978), An assessment of the role of precipitation in magnetospheric convection, J. Geophys. Res., 83, 5227.

Stern, D. P. (1975), The motion of a proton in the equatorial magnetosphere, J. Geophys. Res., 80, 595.

Strobel, D. F., T. R. Young, R. R. Meier, T. P. Coffey, and A. W. Ali (1974), The nighttime ionosphere: $E$ region and lower $F$ region, J. Geophys. Res., 79,3171 .

Sugiura, M., and T. Kamei (1991), Equatorial Dst index 1957-1986, IAGA Bull. 40, Int. Serv. of Geomagn. Indices, Saint-Maur-des-fosses, France. Tsyganenko, N. (2000), Modeling the inner magnetosphere: The asymmetric ring current and Region 2 Birkeland currents revisited, J. Geophys. Res., $105,27,739$
Turner, N. E., D. N. Baker, T. I. Pulkkinen, and R. L. McPherron (2000), Evaluation of the tail current contribution to Dst, J. Geophys. Res., 105, 5431.

Vallat, C., et al. (2004), First comparisons of local ion measurements in the inner magnetosphere with energetic neutral atom magnetospheric image inversions: Cluster-CIS and IMAGE-HENA observations, J. Geophys. Res., 109, A04213, doi:10.1029/2003JA010224.

Volland, H. (1973), A semiempirical model of large-scale magnetospheric electric fields, J. Geophys. Res., 78, 171.

Weimer, D. R. (1996), A flexible, IMF dependent model of high-latitude electric potentials having "space weather" applications, Geophys. Res. Lett., 23, 2549.

Weimer, D. R. (1999), Substorm influence on the ionosphere electric potentials and currents, J. Geophys. Res., 104, 185.

Weimer, D. R., D. M. Ober, N. C. Maynard, M. R. Collier, D. J. McComas, N. F. Ness, C. W. Smith, and J. Watermann (2003), Predicting IMF propagation delay times using the minimum variance technique, J. Geophys. Res., 108(A1), 1026, doi:10.1029/2002JA009405.

Young, D. T., H. Balsiger, and J. Geiss (1982), Correlations of magnetospheric ion composition with geomagnetic and solar activity, J. Geophys. Res., 87, 9077.

P. C. Brandt, R. DeMajistre, D. G. Mitchell, and E. C. Roelof, Johns Hopkins University Applied Physics Laboratory, 11100 Johns Hopkins Road, Laurel, MD 20723-6099, USA.

D. L. Gallagher, National Space Science and Technology Center, NASA Marshall Space Flight Center, 320 Sparkman Drive, Mail Stop XD12, Huntsville, AL 35805, USA.

J. U. Kozyra, M. W. Liemohn, and A. J. Ridley, Department of Atmospheric, Oceanic, and Space Sciences, University of Michigan, 2455 Hayward Street, Ann Arbor, MI 48109-2143, USA. (liemohn@umich.edu)

D. M. Ober, ATK Mission Research, 589 W. Hollis Street, Nashua, NH 03062-1323, USA. 